\title{
Small RNA sequencing of extracellular vesicles identifies circulating miRNAs related to inflammation and oxidative stress in HIV patients
}

\author{
Sukrutha Chettimada', David R. Lorenz ${ }^{1}$, Vikas Misra ${ }^{1}$, Steven M. Wolinsky ${ }^{2}$ and Dana Gabuzda ${ }^{1,3^{*}}$ (D)
}

\begin{abstract}
Background: Extracellular vesicles (EVs) are nano-sized particles secreted by most cells. EVs carry nucleic acids that hold promise as potential biomarkers in various diseases. Human immunodeficiency virus type 1 (HIV) infects CD4+ T cells and induces immune dysfunction, inflammation, and EV secretion, but little is known about EV small RNA cargo in relation to immune dysregulation in HIV-infected individuals. Here, we characterize small RNA carried by circulating EVs in HIV-positive subjects on antiretroviral therapy (ART) relative to uninfected controls by nextgeneration RNA sequencing.

Results: Plasma EVs isolated from HIV-positive and HIV-negative subjects in test $(n=24)$ and validation $(n=16)$ cohorts were characterized by electron microscopy, nanoparticle tracking analysis, and immunoblotting for exosome markers. EVs were more abundant in plasma from HIV-positive compared to HIV-negative subjects. Small RNA sequencing of plasma EVs in the test cohort identified diverse small RNA species including miRNA, piRNA, snRNA, snoRNA, tRNA, and rRNA, with miRNA being the most abundant. A total of 351 different miRNAs were detected in plasma EVs, with the top 50 miRNAs accounting for $90 \%$ of all miRNA reads. miR-26a-5p was the most abundant miRNA, followed by miR-21-5p and miR-148-3p. qRT-PCR analysis showed that six miRNAs (miR-10a-5p, - 21-5p, -27b-3p, - 122-5p, -146a-5p, - 423-5p) were significantly increased in plasma EVs from HIV-positive compared to HIV-negative subjects in the validation cohort. Furthermore, miR-21-5p, -27b-3p, -146a-5p, and - 423-5p correlated positively with metabolite markers of oxidative stress and negatively with anti-inflammatory polyunsaturated fatty acids. Over-representation and pathway enrichment analyses of miRNAs and their target genes predicted functional association with oxidative stress responses, interferon gamma signaling, Toll-like receptor signaling, TGF beta signaling, and Notch signaling.

Conclusions: HIV-positive individuals on ART have increased abundance of circulating EVs carrying diverse small RNAs, with miRNAs being the most abundant. Several miRNAs associated with inflammation and oxidative stress are increased in circulating EVs of HIV-positive individuals, representing potential biomarkers of targetable pathways that contribute to disease pathogenesis.
\end{abstract}

Keywords: Small RNA, MiRNA, Extracellular vesicles, Exosomes, HIV, Inflammation, Oxidative stress

\footnotetext{
* Correspondence: dana_gabuzda@dfci.harvard.edu

'Department of Cancer Immunology and Virology, Dana-Farber Cancer Institute, Boston, MA, USA

${ }^{3}$ Department of Neurology, Harvard Medical School, Boston, MA, USA

Full list of author information is available at the end of the article
}

(c) The Author(s). 2020 Open Access This article is licensed under a Creative Commons Attribution 4.0 International License, which permits use, sharing, adaptation, distribution and reproduction in any medium or format, as long as you give appropriate credit to the original author(s) and the source, provide a link to the Creative Commons licence, and indicate if changes were made. The images or other third party material in this article are included in the article's Creative Commons licence, unless indicated otherwise in a credit line to the material. If material is not included in the article's Creative Commons licence and your intended use is not permitted by statutory regulation or exceeds the permitted use, you will need to obtain permission directly from the copyright holder. To view a copy of this licence, visit http://creativecommons.org/licenses/by/4.0/ The Creative Commons Public Domain Dedication waiver (http://creativecommons.org/publicdomain/zero/1.0/) applies to the data made available in this article, unless otherwise stated in a credit line to the data. 


\section{Background}

Human immunodeficiency virus type 1 (HIV) infection is characterized by progressive decline of $\mathrm{CD} 4+\mathrm{T}$ cell counts, increased immune activation, and inflammation. Although antiretroviral therapy (ART) increases CD4+ T cell counts and improves overall health and life expectancy of HIV-positive individuals, immune activation and chronic inflammation persist. Causes of chronic inflammation in ART-treated HIV patients are incompletely understood, but likely include microbial translocation, elevated expression of type I and II interferons, altered chemokine and cytokine production, and co-infections (e.g., Hepatitis C virus (HCV)) [1-3]. HIV infection and chronic inflammation contribute to generation and accumulation of reactive oxygen species (ROS), compromising antioxidant pathways and leading to oxidative stress, a predictor of morbidity and mortality [4-6]. Identification of biomarkers associated with HIV pathogenesis, inflammation, and oxidative stress is important to gain insights into underlying mechanisms and discover prognostic and diagnostic markers.

Extracellular vesicles (EVs) and their protein and nucleic acid cargo have been extensively studied and used as biomarkers in various diseases including HIV, cancer, cardiovascular diseases, and neurological disorders [7]. EVs, including exosomes $(30-150 \mathrm{~nm})$, microvesicles (MV; microparticles) $(100 \mathrm{~nm}-1 \mu \mathrm{m})$, and apoptotic bodies $(>1 \mu \mathrm{m})$, are secreted by most cell types and have been isolated from plasma and other body fluids. EV cargo includes proteins, lipids, mRNAs, long non-coding RNAs (lncRNA), and several species of small non-coding RNA, such as microRNA (miRNA), Piwi-interacting RNA (piRNA), small nucleolar RNA (snoRNAs), and small nuclear RNA (snRNA), or RNA fragments [8], and provide a means for transfer of RNA between donor and recipient cells. Specific cargo of EVs is dependent on the cell of origin as well as biological conditions such as infection, inflammation, and stress. EVs are involved in cell-to-cell communication and are proposed to play a role in maintaining homeostasis; they have also been implicated in spreading infections via transport of viral and microbial products [7]. EVs, particularly exosomes, can also regulate gene expression by transporting miRNAs to recipient cells and post-transcriptionally controlling translation of corresponding miRNA targets in recipient cells, thereby affecting cellular responses to stress, inflammation, and cell death [9].

Previous studies have shown that HIV-positive individuals have higher abundance of circulating EVs compared to healthy controls [10-12]. EV protein cargo in HIV infection has been studied, revealing enrichment of HIV virulence factors and pro-inflammatory cytokines and chemokines [10, 13-16]. HIV infection induces EV secretion, and EVs from HIV-infected cells transport viral and host components that promote spreading of infection [13-15]. EVs can also inhibit HIV infection by carrying protective factors such as APOBEC3 and interferons $[17,18]$. Additionally, EV RNA exhibiting 5' $5^{\prime}$-triphosphate ends stimulates RIG-I, which induces an interferon response [19]. We previously showed that plasma EVs in ART-treated HIV-positive individuals carry proteins related to immune activation and oxidative stress, and have immunomodulatory effects on myeloid cells, suggesting functional links to inflammation and redox pathways during pathogenesis [12]. Limited studies have explored miRNA content of EVs, showing enrichment of specific miRNAs associated with inflammation and fatal liver disease in HIV-positive individuals $[11,20]$, or lower neuropsychological performance [21]. Little is known about the small RNA repertoire of circulating EVs in HIV-positive individuals. Given that small RNAs are enriched in EVs, we characterized small RNA cargo of plasma EVs in HIV-positive individuals on ART relative to uninfected controls by small RNA sequencing. We then validated some of the mapped miRNAs that are upregulated in treated HIV disease in an independent cohort and examined their association with metabolite markers and pathways related to inflammation and oxidative stress.

\section{Results}

Characteristics of the study cohort

A total of 24 subjects (12 HIV-positive and 12 HIVnegative) and 16 subjects (8 HIV-positive and 8 HIVnegative) subjects comprised the test and validation cohorts, respectively (Fig. 1). Demographic and clinical characteristics are summarized in Table 1 . The median age across cohorts was 54 [interquartile range (IQR): 48 , 50]. The test cohort was comprised of all males $(63 \%$ black), while the validation cohort was $63 \%$ male $(38 \%$ black). All HIV-positive subjects were on ART with suppressed or low plasma viral load. Compared to the test cohort, HIV-positive subjects in the validation cohort had lower median CD4 T cell counts, CD4 nadir, CD4:CD8 ratio, and longer median duration of HIV infection, indicating more advanced HIV disease. Both cohorts had high prevalence of smoking (54 and 56\% in test and validation cohorts, respectively) and cocaine use (50\% in both cohorts). HIV-negative and HIV-positive groups within each cohort were balanced with respect to age, race, HCV status, and cocaine use. Two subjects in the test cohort and no subjects in the validation cohort were $\mathrm{HCV}$-seropositive. All subjects in both cohorts were negative for Hepatitis B virus (HBV) surface antigen and/or DNA.

\section{Characterization of plasma EV fractions}

EV fractions were isolated from HIV-positive $(n=12)$ and HIV-negative $(n=12)$ subjects in the test cohort 


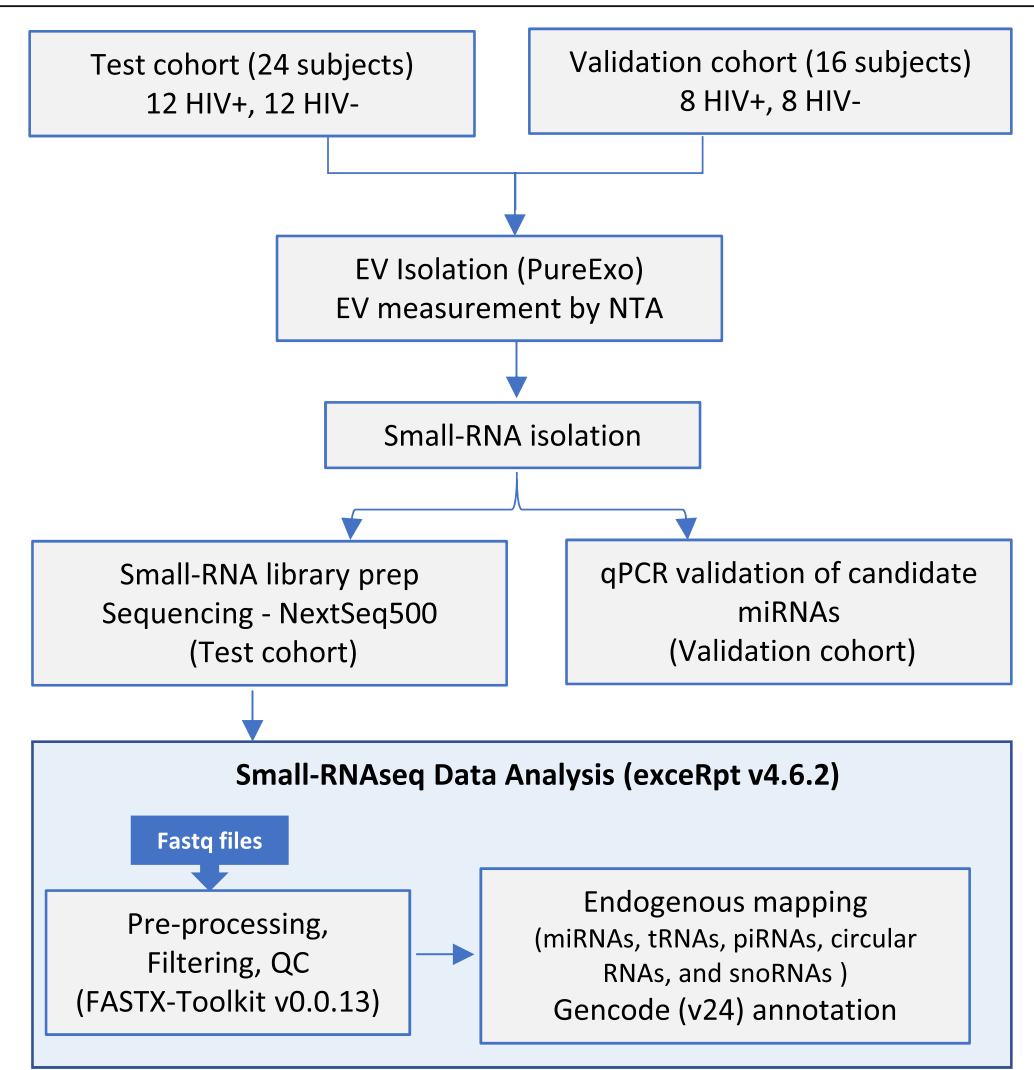

Fig. 1 Study design summarizing the cohort, methodology, and data analysis. $500 \mu$ l plasma from subjects in test or validation cohorts were used to isolate EVs using the PureExo kit. EVs were measured by nanoparticle tracking analysis followed by small RNA isolation. Small RNA libraries were prepared and sequenced on Nextseq500, and data was analyzed using the exceRpt pipeline. Candidate miRNAs were validated by qPCR

Table 1 Clinical and demographic characteristics of test and validation cohorts

\begin{tabular}{|c|c|c|c|c|}
\hline & \multicolumn{2}{|l|}{ Test cohort } & \multicolumn{2}{|l|}{ Validation cohort } \\
\hline & HIV-negative $(n=12)$ & HIV-positive $(n=12)$ & HIV-negative $(n=8)$ & HIV-positive $(n=8)$ \\
\hline $\mathrm{Age}^{a}$ & $52[44-59]$ & $55[45-60]$ & $53[51-54]$ & $57[53-61]$ \\
\hline Black race & $7(58)$ & $8(67)$ & $3(38)$ & $3(38)$ \\
\hline Male gender & $12(100)$ & $12(100)$ & $3(38)$ & $7(88)$ \\
\hline Duration of HIV infection (years) ${ }^{a}$ & & $13[11-20]$ & & 19 [13-22] \\
\hline Viral load (copies/ml) & & $40[10-80]$ & & $40[40-40]$ \\
\hline Viral load $<200$ copies $/ \mathrm{ml}$ & & $11(92)$ & & $8(100)$ \\
\hline 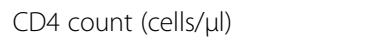 & 955 [776-1142] & 619 [430-734] & & 382 [285-495] \\
\hline CD4 nadir (cells/ul) & $639[539-796]$ & $166[92-257]$ & & $82[26-221]$ \\
\hline CD4/CD8 ratio ${ }^{a}$ & $1.4[1.1-1.9]$ & $0.73[0.54-1.0]$ & & $0.41[0.26-0.51]$ \\
\hline ART use & & $12(100)$ & & $8(100)$ \\
\hline Protease inhibitor use & & $7(58)$ & & $6(75)$ \\
\hline HCV seropositive & $1(8)$ & $1(8)$ & $0(0)$ & $0(0)$ \\
\hline Smoking & $7(58)$ & $6(50)$ & $7(88)$ & $2(25)$ \\
\hline Cocaine use & $6(50)$ & $6(50)$ & $4(50)$ & $4(50)$ \\
\hline
\end{tabular}

Data shown are $\mathrm{n}(\%)$ unless otherwise indicated

${ }^{a}$ median $[\mathrm{IQR}]$ 
using the PureExo exosome isolation kit and characterized by transmission electron microscopy (TEM), nanoparticle tracking analysis (NTA), and Western blotting for exosome markers (Fig. 2). TEM revealed vesicles of $40-70 \mathrm{~nm}$ in diameter (Fig. 2a), consistent with the size range of exosomes. NTA yielded distributions showing that majority of particles were $30-150 \mathrm{~nm}$ diameter, with a peak at 100-120 nm (Fig. 2b). Immunoblotting detected the exosome markers CD9, Flotillin-1, Tsg101, and CD81 in plasma EV fractions, with greater abundance of these markers detected in HIV-positive compared to HIV-negative subjects based on stronger band intensities (Fig. 2c). The endoplasmic reticulum (ER) marker calnexin was not detected by immunoblotting of these plasma EV fractions, suggesting they were free of ER membrane contamination and consistent with results of our previous study [12]. Based on NTA measurements, plasma EVs were more abundant in HIV-positive compared to HIV-negative subjects (mean 5.9 vs. $2.5 \mathrm{X}$ $10^{11}$ particles/ml, respectively, in $30-150 \mathrm{~nm}$ size range; $p=0.001$, Mann-Whitney test), while there was no significant difference in median particle size (114 vs. 116 $\mathrm{nm}$, respectively) (Fig. 2d).
Small RNA classes and distributions in plasma EVs of HIVpositive and HIV-negative subjects

To characterize the plasma EV small RNA repertoire, we sequenced small RNA libraries from all subjects in the test cohort (12 HIV-positive and 12 HIV-negative) and mapped reads to the human genome (hg38) and small RNA databases (Fig. 1). Small RNA was isolated from EV fractions and quality was assessed using the BioAnalyzer platform with a small RNA chip, which showed that the quality of isolated small RNA was comparable between HIV-positive and HIV-negative groups. cDNA libraries were generated and subjected to single-read, 75-bp sequencing generating an average of 22 million reads per library. Raw read data was processed using the exceRpt Small RNA-seq Pipeline [22]. Reads were mapped to human rRNA to exclude rRNA sequences before mapping to the human genome. Size distributions among mapped reads in each sample showed that majority were between $16 \mathrm{nt}$ to $60 \mathrm{nt}$ in length with peaks at $21 \mathrm{nt}$ and $26 \mathrm{nt}$, corresponding to miRNAs and piRNAs, respectively (Fig. 3a).

To examine the diversity of plasma EV RNA cargo, reads were mapped to human genome and small RNA
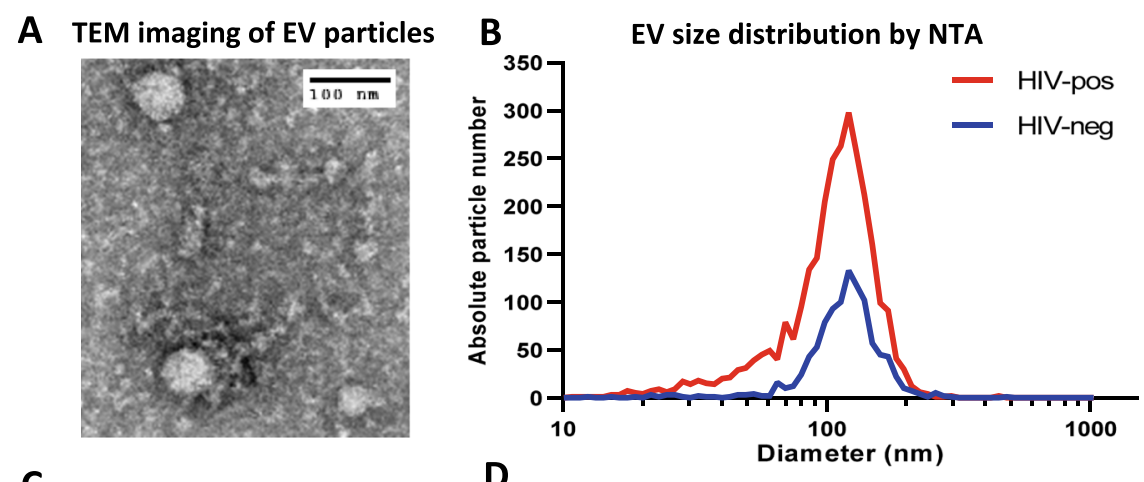

\section{C}
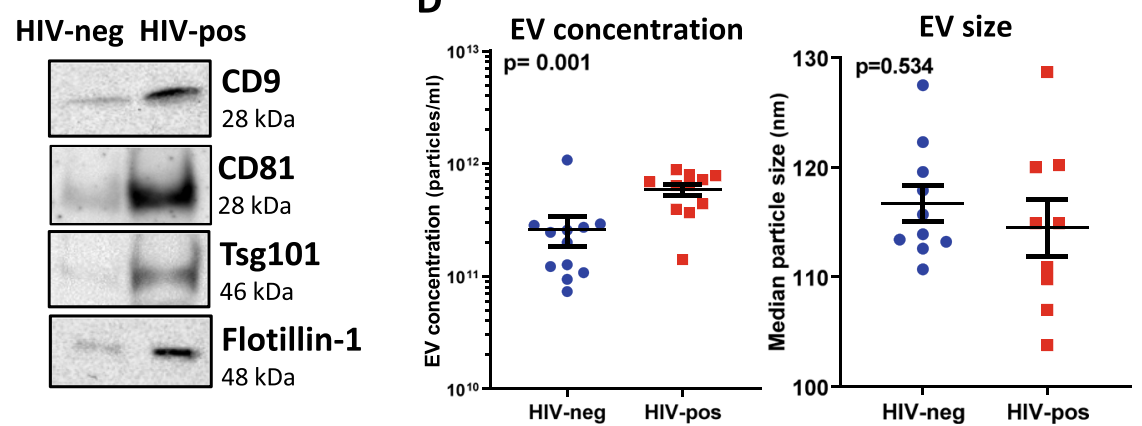

Fig. 2 Characterization of EV fractions isolated from plasma. a. TEM image of isolated EV fraction from a HIV-negative control subject. b. Size distribution of EVs in a representative HIV-negative and HIV-positive subject by nanoparticle tracking analysis (NTA). c. Detection of exosome markers CD9, Flotillin-1, Tsg101, and CD81 by western blotting in pooled HIV-positive $(n=3)$ and HIV-negative $(n=3)$ samples. Three individual HIV-negative subjects were matched for age, gender, race, and cocaine use to three HIV-positive subjects to achieve matching between the pooled samples. Equal amounts of protein $(50 \mu \mathrm{g}$ per lane) were loaded for western blotting; these samples contain exosomal proteins, as well as co-purifying non-exosomal proteins from other sources such as microparticles and residual plasma proteins. Full-length blot images are shown in Supplementary Figure S6. d. EV concentration (left) and median EV size (right) analyzed by NTA in HIV-negative and HIV-positive subjects 
databases for miRNA, piRNA, rRNA, tRNA, circular RNA, and snoRNA. Figure $3 \mathrm{~b}$ shows a comparison of distributions of different RNA species identified in plasma EVs of HIV-negative (left) and HIV-positive (right) subjects in the test cohort. Protein coding reads were the most abundant RNA species in both groups, while miRNA was the most abundant small RNA species. A few subjects had miRNA as the most abundant RNA species instead of protein coding reads, or more snoRNA or tRNA as the most abundant small RNA species instead of miRNA; these findings were not associated with any distinctive subject characteristics. HIVpositive subjects had a higher proportion of protein coding reads compared to HIV-negative subjects (35\% vs. $26 \%$, respectively), and HIV-negative subjects had a higher proportion of miRNA reads compared to HIVpositive subjects ( $19 \%$ vs. $13 \%$, respectively) $(p<0.0001$, chi-square test). The next major classes of small RNAs detected were small nuclear RNA (snRNA), transfer RNA (tRNA), antisense RNA, long intergenic noncoding RNA (lincRNA), ribosomal RNA (rRNA), piRNA, small nucleolar RNA (snoRNA), and miscellaneous RNA (RNAs mapped to human genome, but not to any known RNA species in human genome). Supplementary Table 1 shows the complete list and total counts of each RNA species mapped across all samples. Number of uniquely mapped RNAs of different species is shown in Fig. $3 \mathrm{c}$ and summed read counts for each species are shown in Supplementary Figure S1. 26 and 35\% of protein coding reads (Fig. 3b) mapped to 11,051 and 11, 617 unique protein coding species in HIV-negative and HIV-positive subjects, respectively. Nineteen percent and $13 \%$ of miRNA reads mapped to 313 and 226 unique miRNAs in HIV-negative and HIV-positive subjects, respectively (Supplementary Table 2).

We identified a total of 14,500 unique protein coding sequences; among these, 392 (top 2.7\%) had counts > 10 in at least $25 \%$ of the samples and accounted for $60 \%$ of all protein coding reads. Although fragments derived from protein coding sequences were the most abundant RNA species in terms of overall reads and number of different protein coding sequences, differential expression analysis of these protein coding sequences for the top 70 genes (filtered for genes with counts $>10$ in at least $25 \%$ samples and not identified in blank samples) did not reveal any significantly altered protein coding sequences in HIVpositive vs. HIV-negative subjects after correction for multiple testing (Benjamini-Hochberg FDR adjusted $p$-value $>0.1$ ) (Supplementary Table 3). The most abundant protein coding sequence reads were NPFFR1 (Neuropeptide FF Receptor 1), which is associated with G proteincoupled receptor activity and neuropeptide receptor activity, followed by WDR74 (WD Repeat Domain 74), a regulatory protein of the MTREX-exosome complex. Protein coding sequences for STEAP4 (STEAP4 Metalloreductase) and UTRN (Utrophin) showed an increasing trend in HIV-positive compared to HIV-negative subjects that did not reach significance (FDR $>0.1$ ). Small nucleolar RNAs

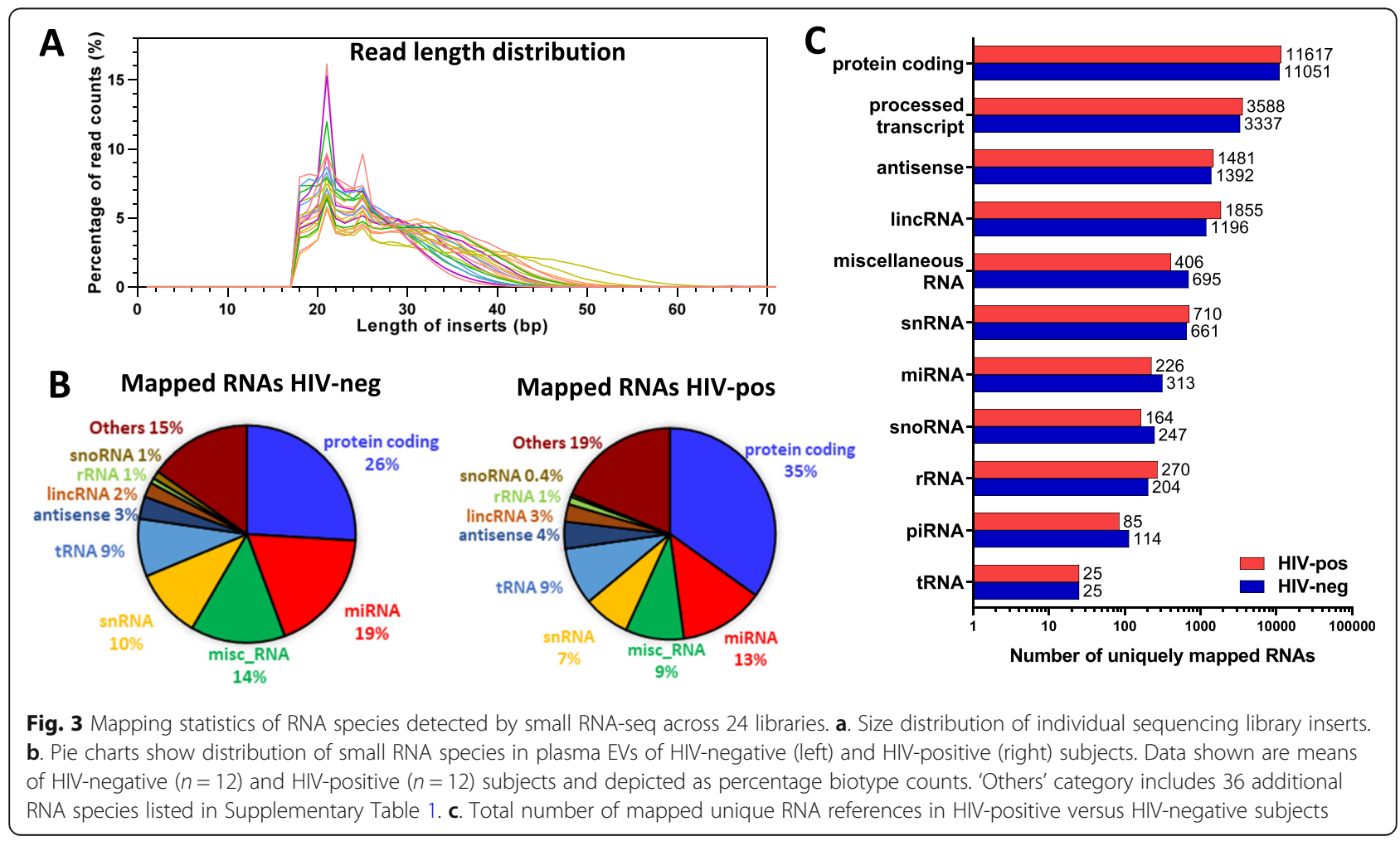


SNORD104, SNORD2, SNORD69, and SNORD63 were the most abundant snoRNAs, accounting for $50 \%$ of all snoRNA reads. Small nuclear RNAs U2, RNU1 and RNU2 were the predominant snRNAs, and were detected in majority of samples. PiRNA was the least abundant type of small RNA detected, with hsa-piR-018780 being the most abundant; however, majority of samples had counts $<10$ for this piRNA. Mapping to the tRNA database identified 25 distinct tRNA species; among these tRNA ${ }^{\text {Gly }}(21 \%)$ and tRNA ${ }^{\text {Glu }}(20 \%)$ were the predominant tRNAs and were detected in all samples, while none of the remaining tRNAs exceeded $9 \%$ of total tRNA counts. These results show that circulating EVs in HIV-positive and HIV-negative subjects carry diverse small RNA species including miRNA, piRNA, snRNA, tRNA, and rRNA, with miRNAs being the most abundant small RNA species.

\section{Analysis of miRNA profiles in circulating EVs}

A total of 351 unique miRNAs were identified (Fig. 4a and Supplementary Table 2). The top 50 most abundant miRNAs accounted for $90 \%$ of all miRNA reads (aqua bars and embedded graph). miR-26a-5p was the most abundant miRNA, followed by miR-21-5p and miR-148-3p. HIVpositive and HIV-negative subjects had similar distributions of number of unique miRNAs detected (Fig. $4 \mathrm{~b}, p=0.89$, Mann-Whitney test). To identify outliers, we performed principal component analysis (PCA) on the top 50 most abundant miRNAs. PCA analysis revealed 3 outliers (HIVnegative) (Supplementary Figure S2) that were excluded from downstream analyses, along with one sample with low number of identified miRNAs ( 0 count for $>94 \%$ of miRNAs). PCA analysis after outlier exclusion did not reveal distinct clusters differentiating HIV-positive and HIVnegative subjects (Fig. 4c). Additionally, there were no obvious outliers or clusters associated with age, race, cocaine, or smoking. Differential expression analysis of the top $50 \mathrm{miR}-$ NAs in HIV-positive vs. HIV-negative subjects is shown in Table 2. No miRNAs were significantly upregulated in HIV-positive compared to HIV-negative subjects by DEseq2 analysis after correction for multiple testing (FDR < 0.1 ), while only one miRNA (miR-181a-5p) was significantly downregulated $(\log 2 \mathrm{FC}=-5.88$, FDR-adjusted $p$ value $=0.0008$ ). Two HCV-positive samples $(\mathrm{HIVn}-7$ and HIVp-10) did not have distinctive miRNA profiles compared with HCV-negative samples in Supplementary Table 2 , and analyzing the data after removing these two samples did not significantly alter the main findings in Table 2.

miRNAs associated with HIV infection, inflammation, and oxidative stress are increased in plasma EVs of HIVpositive compared with HIV-negative subjects

We selected 8 miRNAs identified by small RNAsequencing (miR-27b-3p, - 21-5p, -125b-5p, - 122-5p,
$-10 a-5 p,-423-5 p,-146 a-5 p$ and let-7a-5p) for qRTPCR validation based on the following criteria: an increasing trend in HIV-positive compared to HIVnegative by DEseq2 analysis (miR-10a-5p, - 122-5p, $-146 a-5 p,-27 b-3 p,-423-5 p$, and let-7a-5p), and/or previously shown to be associated with HIV infection (miR122-5p, -125b-5p, -146a-5p, - 21-5p, -27b-3p, and 423-5p) [23-27], or inflammation and oxidative stress (miR-10a-5p, -125b-5p, -146a-5p, -21-5p, and -27b3p) $[28-31]$.

To confirm the identity of specific miRNAs detected by small RNA-seq, we performed manual alignment of miRNA reads using the CodonCode Aligner tool. To accomplish this, all reads from a sample mapping to a specific miRNA were manually aligned to the original miRNA stem-loop sequence from miRbase v21. Representative alignments for miR-27b-3p and miR-146a-5p from HIV-positive and negative samples are shown in Fig. 5, which shows that $100 \%$ of reads mapped to miR$146 a-5 p$ with 1 or 0 nucleotide mismatch and $>95 \%$ of reads mapped to miR-27b-3p with 1 or 0 nucleotide mismatch, suggesting these miRNAs and their isoforms (isomiRs) [32] are indeed present in these plasma EV samples.

The miRNAs in plasma are associated with lipoproteins (LDL/HDL) and ribonucleoproteins, which protect extracellular RNAs against RNase-mediated degradation and can be coprecipitated during EV isolation [33-35]. To exclude extra-vesicular miRNAs, EVs were isolated using the PureExo exosome isolation kit with the following modification: Proteinase $\mathrm{K}$ was added to plasma samples to release protein-associated miRNAs followed by RNase A treatment to degrade extravesicular RNAs. Proteinase $\mathrm{K}$ and RNase treatment resulted in reduction of small particles $(10-30 \mathrm{~nm})$ corresponding to lipoprotein particles as seen by TEM, particle size distribution, and particle concentration measurements (Supplementary Figure S3). Elimination of lipoprotein particles was further confirmed by Western blotting for ApoA1 (major structural protein component of HDL), soluble exosome markers (Alix and Tsg101), and membrane exosome markers (CD81- outer membrane and Flotillin-1 inner membrane). Proteinase $\mathrm{K}$ and RNase treatment degraded extra-exosomal proteins, while retaining intraexosomal cargo as indicated by absence of CD81 and ApoA1 bands, and retention of Flotillin-1, Alix, and Tsg101 bands (Supplementary Figure S3).

To validate selected miRNAs in plasma EVs by qRTPCR, we isolated small RNA from EV fractions of 8 HIV-positive and $8 \mathrm{HIV}$-negative subjects from the validation cohort. EV small RNA was isolated from equal plasma volumes and equal volumes of RNA were loaded for validation of candidate miRNAs by qRT-PCR analysis. Analysis of plasma EV fractions by NTA showed 

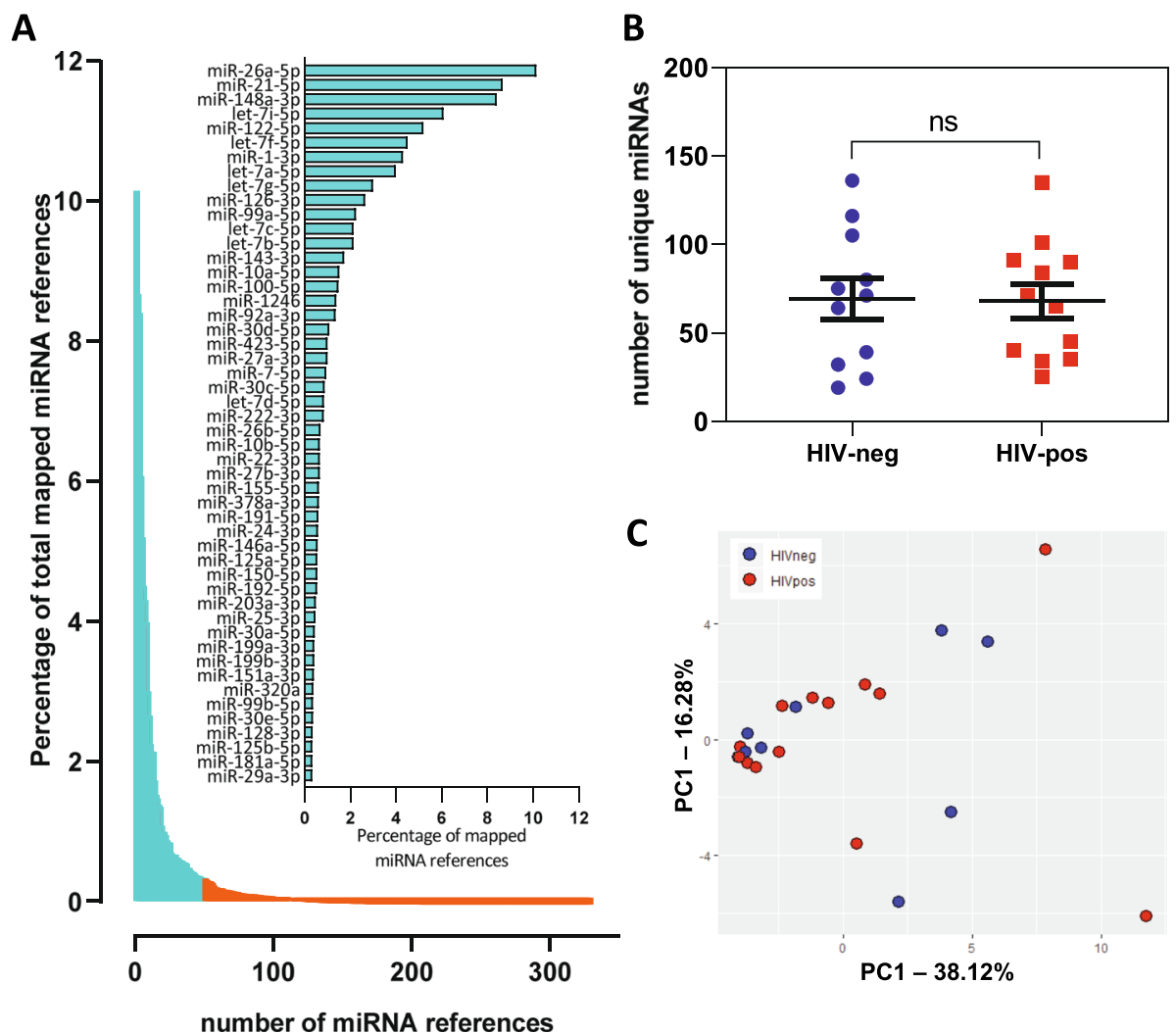

Fig. 4 Small RNA-seq miRNA profiles in test cohort HIV-positive and HIV-negative subjects. a. Percentage (sorted from high to low) of each detected mature miRNA in all mapped miRNA reads in 24 subjects. The top 50 miRNAs (90\%) are highlighted in aqua and shown in the embedded graph. $\mathbf{b}$. Distribution of number of unique miRNAs in HIV-negative and HIV-positive subjects ( $p=0.89$, Mann-Whitney test). $\mathbf{c}$. Principal component analysis of top 50 miRNAs in HIV-positive (red, $n=12$ ) and HIV-negative subjects (blue, $n=8$ )

that HIV-positive subjects had higher EV numbers compared to HIV-negative subjects (Fig. 6a, $p=0.081$ ), while EV size distribution was similar between groups. We detected significantly higher levels of miR-27b-3p, - 21-5p, $-122-5 p,-10 a-5 p,-146 a-5 p$, and $-423-5 p$ in plasma EVs from HIV-positive compared to HIV-negative subjects by qRT-PCR (Fig. 6 b, $p<0.05$, Mann-Whitney test), whereas miR-125b-5p and let-7a-5p did not show a significant difference ( $p=0.24$ and 0.067 , respectively). As an additional control for qRT-PCR validation, we tested miR-7-5p, which showed no significant difference in plasma EVs from HIV-positive compared to HIVnegative subjects $(p=0.112)$, consistent with results in Table $2(p=0.991)$. Unsupervised hierarchical clustering in heatmaps show that significantly altered miRNAs (miR-27b-3p, - 21-5p, - 122-5p, -10a-5p, -146a-5p, and - 423-5p) distinguished between HIV-positive and HIV negative subjects based on z-scored qRT-PCR measurements (Fig. 6c). Given that cocaine abuse promotes oxidative stress and inflammation in HIV-positive subjects, we compared levels of these miRNAs between HIV-positive and HIV-negative subjects stratified by cocaine use. These miRNAs remained elevated in HIV-positive cocaine users and non-users compared to HIV-negative cocaine users and non-users, respectively (Supplementary Figure S4). In contrast, there was no change in miRNA levels associated with cocaine use within the HIV-positive or HIV-negative groups. Thus, the observed changes in plasma EV miRNA 
Table 2 Differential expression analysis of top 50 miRNAs in HIV-positive $(n=12)$ versus HIV-negative $(n=8)$ subjects from the test cohort

\begin{tabular}{|c|c|c|c|c|}
\hline & baseMean & $\log 2 \mathrm{FC}$ & $P$ value & $P$ adj \\
\hline hsa-miR-26a-5p & 620 & -0.106 & 0.8216 & 0.9831 \\
\hline hsa-miR-148a-3p & 514.38 & 0.798 & 0.2256 & 0.6636 \\
\hline hsa-miR-21-5p* & 493.5 & -0.405 & 0.4709 & 0.981 \\
\hline hsa-let-7i-5p & 318.91 & -0.191 & 0.7614 & 0.9831 \\
\hline hsa-miR-122-5p* & 288.3 & 0.953 & 0.4941 & 0.9831 \\
\hline hsa-miR-1-3p & 243.23 & 0.662 & 0.6196 & 0.9831 \\
\hline hsa-let-7a-5p* & 224.95 & 1.512 & 0.0699 & 0.3493 \\
\hline hsa-let-7f-5p & 216.84 & -1.594 & 0.1263 & 0.4859 \\
\hline hsa-miR-423-5p* & 210.36 & 0.239 & 0.8054 & 0.9831 \\
\hline hsa-let-7b-5p & 181.67 & 1.098 & 0.2963 & 0.7134 \\
\hline hsa-miR-126-3p & 134.04 & 3.067 & 0.0056 & 0.1169 \\
\hline hsa-let-7 g-5p & 113.18 & -0.108 & 0.9161 & 0.9831 \\
\hline hsa-let-7c-5p & 109.04 & 2.39 & 0.0466 & 0.259 \\
\hline hsa-miR-30d-5p & 102.88 & -1.677 & 0.2039 & 0.6371 \\
\hline hsa-miR-99a-5p & 96.3 & 0.527 & 0.6449 & 0.9831 \\
\hline hsa-miR-92a-3p & 86.99 & 0.779 & 0.4643 & 0.981 \\
\hline hsa-miR-10a-5p* & 83 & 2.9 & 0.0213 & 0.1779 \\
\hline hsa-miR-100-5p & 80.05 & 1.389 & 0.2811 & 0.7134 \\
\hline hsa-miR-22-3p & 79.59 & 2.307 & 0.0898 & 0.3743 \\
\hline hsa-miR-143-3p & 73.61 & 1.308 & 0.3139 & 0.7134 \\
\hline hsa-miR-486-5p & 69.46 & 1.373 & 0.3024 & 0.7134 \\
\hline hsa-miR-1246 & 64.35 & -2.662 & 0.0342 & 0.2441 \\
\hline hsa-miR-155-5p & 64.01 & -0.322 & 0.7998 & 0.9831 \\
\hline hsa-miR-151a-3p & 59.85 & -0.261 & 0.8725 & 0.9831 \\
\hline hsa-miR-7-5p & 57.03 & 0.038 & 0.9751 & 0.9917 \\
\hline hsa-miR-99b-5p & 51.54 & -0.933 & 0.7672 & 0.9831 \\
\hline hsa-miR-27a-3p & 50.45 & 1.684 & 0.1728 & 0.5761 \\
\hline hsa-miR-320a & 50.4 & 0.352 & 0.811 & 0.9831 \\
\hline hsa-miR-30c-5p & 49.92 & -1.558 & 0.2631 & 0.7134 \\
\hline hsa-let-7d-5p & 44.98 & 2.287 & 0.0391 & 0.2447 \\
\hline hsa-miR-101-3p & 44.39 & -0.6 & 0.674 & 0.9831 \\
\hline hsa-miR-192-5p & 41.22 & 2.429 & 0.0795 & 0.3615 \\
\hline hsa-miR-222-3p & 41.18 & 0.194 & 0.8841 & 0.9831 \\
\hline hsa-miR-199a-3p & 40.5 & 0.08 & 0.9438 & 0.9831 \\
\hline hsa-miR-199b-3p & 40.5 & 0.08 & 0.9438 & 0.9831 \\
\hline hsa-miR-10b-5p & 34.17 & 0.197 & 0.8896 & 0.9831 \\
\hline hsa-miR-181a-5p & 33.66 & -5.884 & $1.59 \mathrm{E}-05$ & 0.0008 \\
\hline hsa-miR-27b-3p* & 31.04 & 2.583 & 0.0112 & 0.1169 \\
\hline hsa-miR-26b-5p & 30.29 & 0.012 & 0.9917 & 0.9917 \\
\hline hsa-miR-378a-3p & 29.32 & 3.691 & 0.0117 & 0.1169 \\
\hline hsa-miR-24-3p & 28.94 & 1.696 & 0.1589 & 0.5674 \\
\hline hsa-miR-191-5p & 28.19 & -0.71 & 0.676 & 0.9831 \\
\hline hsa-miR-25-3p & 25.4 & 0.235 & 0.8851 & 0.9831 \\
\hline
\end{tabular}


Table 2 Differential expression analysis of top 50 miRNAs in HIV-positive $(n=12)$ versus HIV-negative $(n=8)$ subjects from the test cohort (Continued)

\begin{tabular}{lllll}
\hline & baseMean & Log2 FC & $P$ value & P adj \\
\hline hsa-miR-125a-5p & 22.86 & -4.3 & $\mathbf{0 . 0 1 0 4}$ & 0.1169 \\
hsa-miR-451a & 22.8 & 0.443 & 0.7961 & 0.9831 \\
hsa-miR-146a-5p* & 22.33 & 0.856 & 0.5596 & 0.9831 \\
hsa-miR-125b-5p* & 14.62 & -0.665 & 0.6723 & 0.9831 \\
hsa-miR-218-5p & 14.38 & 1.004 & 0.5147 & 0.9831 \\
hsa-miR-423-3p & 12.6 & 0.485 & 0.7753 & 0.9831 \\
hsa-miR-30a-5p & 10.24 & -0.284 & 0.8542 & 0.9831 \\
\hline
\end{tabular}

* miRNAs selected for qRT-PCR validation are indicated with an asterisk. Significant raw $p$-values are in bold

levels were likely related to effects of HIV infection rather than cocaine use.

\section{Functional over-representation analysis of miRNAs}

We performed functional over-representation analysis (ORA) of differentially expressed miRNAs identified in the validation cohort using the web-based application miEAA (miRNA Enrichment Analysis and Annotation). ORA of miRNAs: miR-27b-3p, -21-5p, -122-5p, -10a5p, $-146 a-5 p$, and $-423-5 p$ found significant enrichment of categories: Pathways (176 terms), Gene Ontology (GO, 732 terms), and Diseases (7 terms). ORA results are shown in Supplementary Table 4; HIV-positive versus HIV-negative subjects had significant enrichment of miRNA targets mapping to pathways such as oxidative stress response, interferon gamma signaling, Toll-like receptor signaling, $\mathrm{T}$ cell activation, TGF beta signaling, and Notch signaling among the top 20 most significant pathways $(p=0.008)$. Among the top $25 \mathrm{GO}$ terms enriched were response to stress, positive regulation of $\mathrm{T}$ cell cytokine production, cytoplasmic membrane bound vesicle, microtubule-based movement, late endosome, and endocytosis $(p \leq 0.008)$. Furthermore, $\geq 5$ of 6 up-regulated miRNAs supported these ORA results ('observed' column in Supplementary Table 4). Disease terms enriched by ORA were autoimmune diseases, inflammation, metabolic diseases, and obesity $(p<0.05)$. These results suggest that miRNAs associated with factors related to HIV infection and EV secretion are enriched in plasma EVs of HIV-positive subjects.

\section{miRNA-target enrichment and functional analysis}

Target genes of differentially expressed miRNAs identified in the validation cohort (miR-27b-3p, - 21-5p, - 122-5p, $-10 a-5 p,-146 a-5 p$, and $-423-5 p)$ were predicted using predictor algorithms queried by miRDIP v4.1.11.1 and miRWalk v3.0, along with experimentally validated targets deposited in miRTarbase v7.0. The complex interaction relationship between these 6 miRNAs and their target genes was visualized as a network (Fig. 6d) for a set of 61 predicted and validated targets (Supplementary Table 5). miR-21-5p and miR-27b-3p showed the highest number of overall target genes $(n=33$ genes each), followed by miR146a-5p and miR-122-5p. Majority of these target genes were associated with more than one miRNA (36 genes, orange nodes). For example, NFAT5 is a predicted target of 5 of the 6 miRNAs, while DDHA1 is a predicted target of 4 of the 6 miRNAs. We performed functional enrichment analysis of the miRNAtarget genes with REACTOME pathways using the ClueGO v2.5.5 plugin of Cytoscape 3.7.2 (Fig. 6e). A set of 31 statistically significant pathways $(p<0.005)$ were identified including Toll-like receptor signaling cascade, MyD88 cascade, IRAK1 recruits IKK complex, TGF-beta receptor signaling, Notch signaling pathway, and NOD1/2 signaling (Supplementary Table 6), in accordance with the over-representation analysis (Supplementary Table 4). TNF receptorassociated factor 6 (TRAF6) and IL-1 receptor associated kinase (IRAK1) genes were associated with majority of these pathway terms; both genes are predicted and validated targets of miR-146a-5p.

\section{EV miRNAs correlate with oxidative stress markers}

We previously showed positive correlation of oxidative stress metabolites (methionine and cysteine metabolism) and kynurenine:tryptophan ratio (K:T ratio, immune activation marker), and negative correlation of antiinflammatory polyunsaturated fatty acid (PUFA) metabolites ( $\mathrm{n}-3$ and $\mathrm{n}-6$ PUFA metabolism) with exosome markers [12]. Based on these findings, we performed untargeted metabolomic profiling of plasma from subjects in the validation cohort using the platform described in [12], which detected 655 endogenous metabolites from which we selected 12 metabolites or ratios (Supplementary Table 7) related to: 1) tryptophan catabolism (increased with immune activation) [36, 37]; 2) methionine and cysteine metabolism (altered with oxidative stress) [38, 39]; and 3) n-3 and n-6 PUFA metabolism (anti-inflammatory pathway). Metabolite 


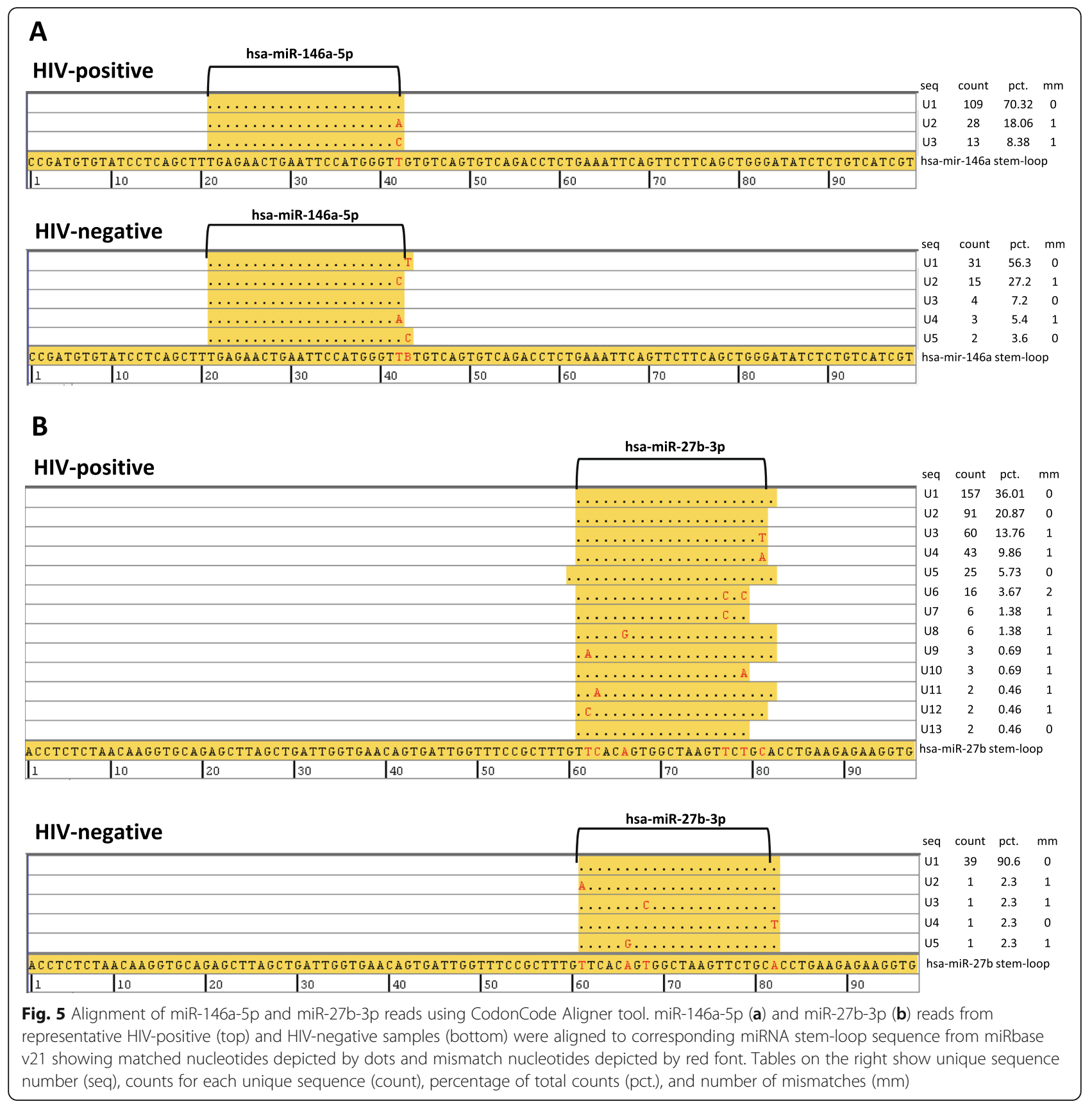

markers of oxidative stress (cysteine, cystine, cysteine ssulfate, cysteinyl glycine, N1-methyladenosine; $p<0.05$, Mann-Whitney test) and $\mathrm{K}: \mathrm{T}$ ratio $(p=0.08)$ were increased in HIV-positive compared to HIV-negative subjects; two additional markers of oxidative stress (cysteinyl glycine, oxidized and methionine sulfone) showed similar trends (Fig. 7a). Given greater abundance of miR-27b-3p, - 21-5p, -146a-5p, and - 423-5p in HIVpositive vs. control subjects in the validation cohort, we examined relationships between these miRNAs and plasma metabolite markers of oxidative stress, immune activation, and EV abundance in HIV-positive and HIV- negative subjects. Increased EV numbers in HIV-positive subjects correlated positively with miR-27b-3p, $-21-5 p$, $-146 a-5 p$, and $-423-5$ p (Fig. 7 b, $p<0.05$ ). Metabolites of the cysteine metabolism pathway (i.e. cysteine, cystine, oxidized cys-gly, cysteine s-sulfate) correlated positively (Fig. 7c), while PUFA metabolites (docosahexaenoate (22:6n3) (DHA), n-3 (22:5n3) and n-6 (22:5n6) docosapentaenoate (DPA), and eicosapentaenoate (20:5n3) (EPA)) correlated negatively with each of the 4 miRNAs tested (Supplementary Figure S5). The immune activation marker $\mathrm{K}: \mathrm{T}$ ratio did not correlate with any of the miRNAs tested (data not shown). We also tested 


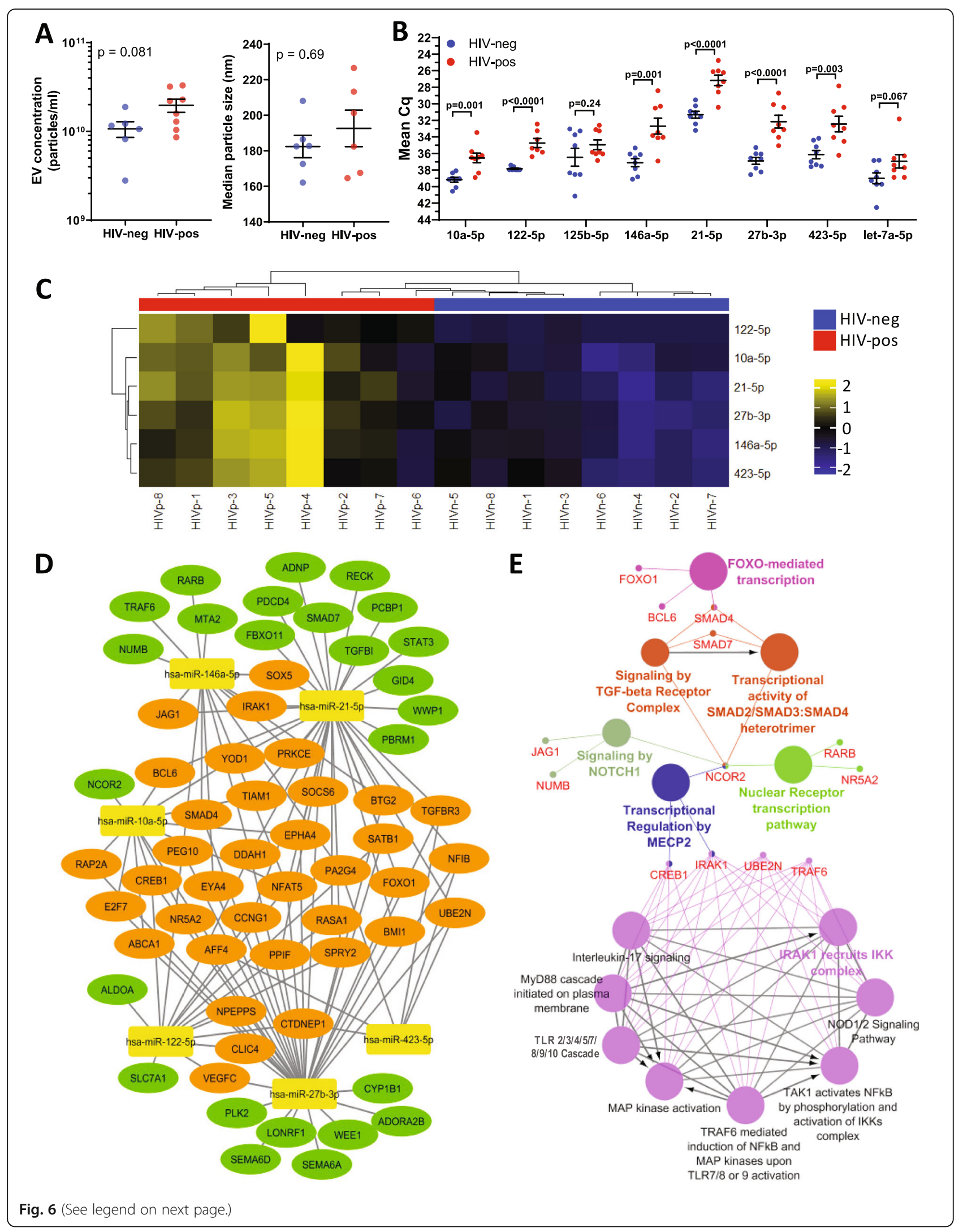




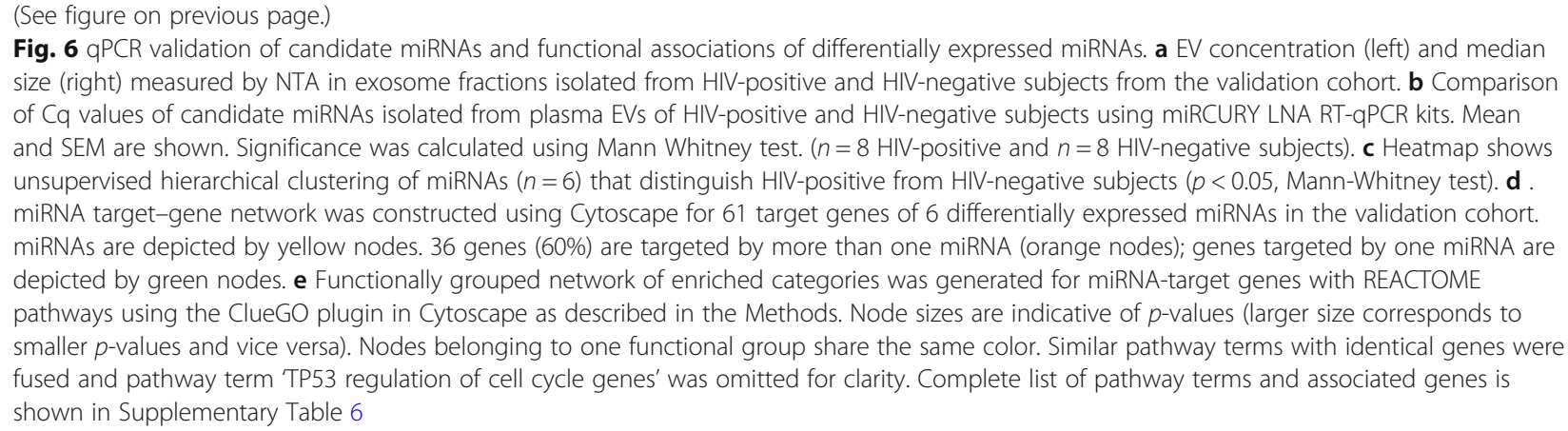

correlation of miRNAs miR-10a-5p and miR-122-5p with these metabolites, but did not find any significant correlations (data not shown).

\section{Discussion}

In this study, we applied next generation small RNA sequencing to characterize RNA cargo of plasma EVs in HIV-positive subjects on ART relative to healthy controls and evaluated abundance of different RNA species and association of miRNAs with disease-related factors. To our knowledge, this is the first study exploring EV small RNA cargo in treated HIV patients. We found higher abundance of plasma EVs in treated HIV-positive subjects compared to HIV-negative controls, consistent with our previous study [12]. Small RNA sequencing of libraries from plasma EVs showed similar sequencing depth between HIV-positive and HIV-negative subjects (23 versus 21.8 million average reads, respectively). Mapping to the human genome and small RNA databases revealed distributions of diverse coding and non-coding RNA species including protein coding sequences, miRNA, rRNA, tRNA, lincRNA, snRNA, and snoRNA, with protein coding sequences being the most abundant RNA species and miRNA being the most abundant small RNA. Consistent with our findings, a previous study of plasma EVs from healthy volunteers identified protein coding sequences as the most abundant RNA species [40]. Yuan $\mathrm{T}$ et al. reported miRNAs as the most abundant plasma extracellular RNA species followed by piRNAs in a study of cancer patients [41], whereas piRNA was among the least abundant RNA species in our study. Based on detection of mostly exosome-sized vesicles in isolated $\mathrm{EV}$ fractions together with prior studies showing miRNAs enrichment in exosomes, EV miRNAs characterized in our study most likely reflect exosomal miRNAs.

miRNAs are abundant in biofluids and widely studied as biomarkers of diseases and biological processes. We detected a total of 351 different miRNAs in plasma EVs, with the top 50 miRNAs accounting for $90 \%$ of all miRNA reads. miR-26a-5p was the most abundant
miRNA, followed by miR-21-5p and miR-148-3p, consistent with another report studying small RNA signatures in different body fluids where miR-26a-5p and miR-21-5p were the most abundant miRNAs in plasma [42]. Liver specific miRNAs miR-122 and miR-22 were among the top 50 miRNAs identified in the test cohort, suggesting liver is one potential source. miR-122 showed an increasing trend in the test cohort and was significantly elevated in HIV-positive subjects in the validation cohort. Elevated miR-122 is implicated in viral hepatitis and contributes to hepatotoxicity in HIV-positive individuals on ART $[20,43,44]$. Other abundant sources of miRNAs in plasma are LDL and HDL particles. While LDL-miRNA profiles align more closely with EVmiRNAs, HDL-miRNAs have distinct miRNA profiles [35]. Importantly, miR-135a-3p, an HDL-associated miRNA, was not among the top 50 miRNAs detected in our samples. Likewise, a platelet-associated miRNA, miR-223, was not detected in majority of samples (mean count $<5$ ), suggesting platelets were not a major source of detected miRNAs. The diversity of miRNA species was similar in HIV-positive and HIV-negative subjects (Fig. 4b), and we did not detect any significantly altered miRNAs in HIV-positive versus HIV-negative subjects by DEseq2-differential expression analysis of the top 50 miRNAs. The inability of DEseq2 to detect significantly altered miRNAs in our study could be due to the low RNA input from small volume of plasma used to isolate EV fractions, and/or PCR bias during library preparation.

We selected miRNAs showing an increasing trend in HIV-positive subjects in the test cohort, or previously linked to HIV infection, inflammation, and/or oxidative stress, for qRT-PCR validation in an independent cohort, and confirmed the identity of selected miRNAs by aligning reads to their corresponding stem-loop sequence from miRbase. Six miRNAs quantified by qRT-PCR (miR-27b-3p, - 21-5p, - 122-5p, -10a-5p, - 423-5p, and -146a-5p) were increased in HIV-positive compared to HIV-negative subjects in the validation cohort. Possible reasons why these six miRNAs were significantly increased in the validation cohort, while only two of these 


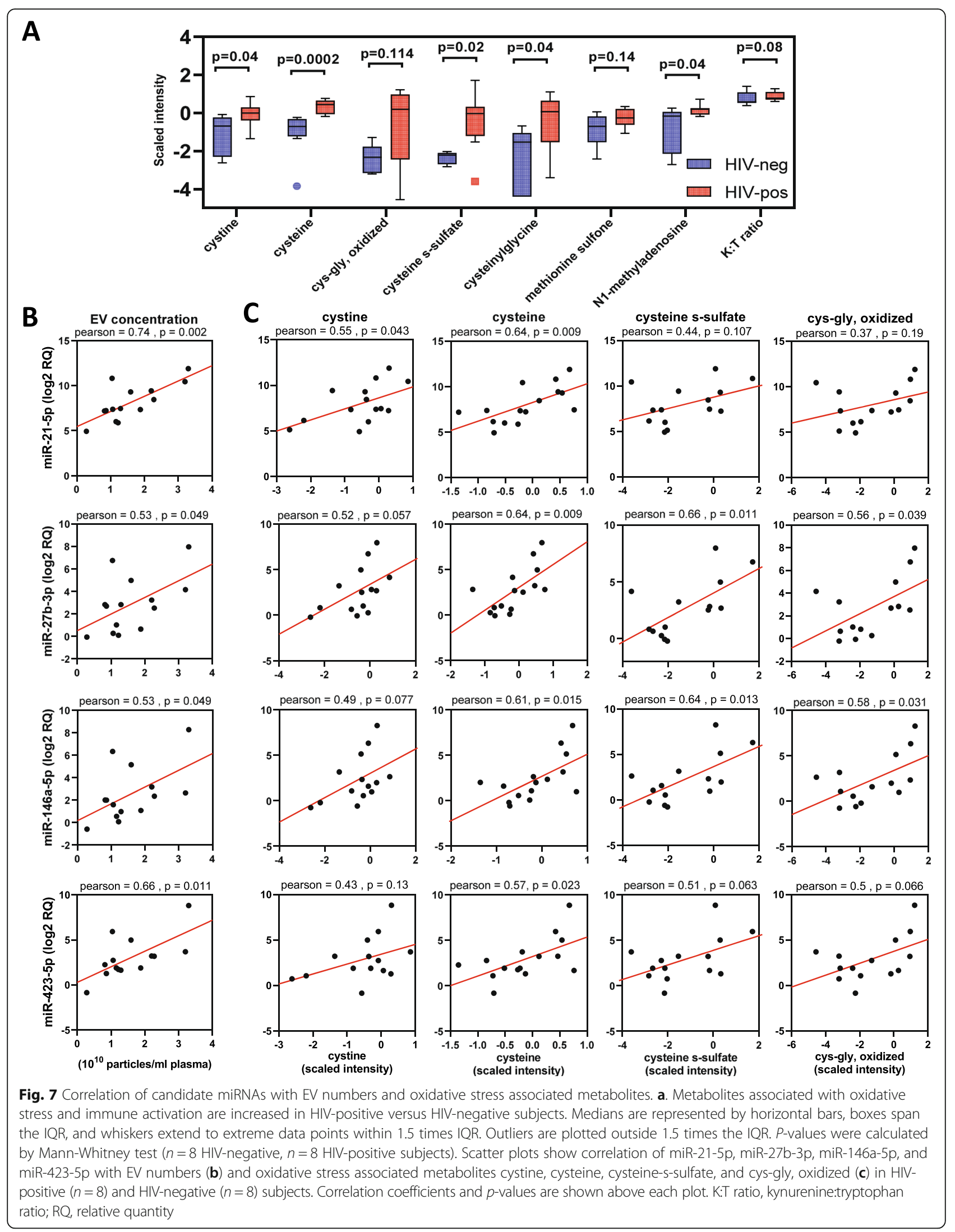


(miR-27b-3p and -10a-5p) had a statistically significant increase (unadjusted $p<0.05$ ) in the test cohort, include the following: 1) validation cohort consisted of HIVpositive subjects with more advanced HIV disease compared to the test cohort; 2) higher specificity of targeted qRT-PCR as compared to whole library amplification during RNA-seq; and 3) normalization was performed for the test cohort during differential expression analysis by DEseq2, while Cq values from qRT-PCR were directly compared in the validation cohort for equal volumes of starting plasma and EV RNA.

Among the six miRNAs elevated in plasma EVs of HIV-positive subjects in the validation cohort, miR125b-5p, -146a-5p, - 21-5p, -27b-3p, and - 423-5p were previously associated with HIV infection. miR-125b-5p may play a role in HIV latency [25], while miR-146a is elevated in plasma of HIV-positive subjects, and may inhibit HIV by disrupting RNA-mediated Gag assembly and virion budding $[24,45]$. Elevated levels of miR-27b decreased viral gene expression levels of HIV in vitro [23]. Thus, increased levels of these miRNAs may play a protective role during HIV infection. miR-27b and other miRNAs including miR-10a, $-21,-125 \mathrm{~b}$, and $-146 \mathrm{a}$ are increased in vascular tissues during inflammation and oxidative stress [28]. One factor that can promote inflammation and oxidative stress in HIV-positive subjects is cocaine abuse [46, 47], which was prevalent in both the test and validation cohorts (50\%). However, we did not detect significantly altered miRNAs with cocaine use in HIV-positive or healthy control groups in either cohort.

Our previous studies identified changes in plasma metabolites related to chronic immune activation, oxidative stress, and inflammation in HIV patients on ART [12]. Oxidative stress metabolites correlated positively, while anti-inflammatory PUFA metabolites correlated negatively, with exosome markers. Oxidative stress increases exosome secretion in vitro, which can communicate protective messages to other cells in part by exosomal shuttling of RNA cargo [48]. EV-associated miRNAs, miR27b-3p, - 21-5p, -146a-5p, and - 423-5p were increased in HIV-positive subjects compared to HIV-negative controls and correlated positively with metabolites associated with oxidative stress (cysteine, cystine, oxidized cysgly, cysteine s-sulfate). miRNAs miR-27b and miR-21 are increased in response to oxidative stress in macrophages and regulate macrophage functions via the NFkB pathway [49], while miR-423 targets the HIV genome in Gag regions and may interfere with HIV replication [50]. Our finding that oxidative stress- and HIVassociated miRNAs within plasma EVs correlate positively with oxidative stress metabolites is consistent with a functional role for exosomes in modulating HIV pathogenesis and redox homeostasis.
Over-representation and pathway enrichment analysis of differentially expressed miRNAs and their target genes predicted functional associations of these upregulated EV miRNAs with oxidative stress response, interferon gamma signaling, Toll-like receptor signaling, TGF beta signaling, and Notch signaling. Interestingly, our previous report investigating the EV proteome identified Notch4 in plasma EVs and showed that EV-associated Notch4 was increased in HIV-positive individuals and correlated with immune activation markers [12]. Majority of terms in pathway enrichment analysis were associated with TRAF6 and IRAK1 genes, which are targeted by miR-146a-5p [51]. miR-146a-5p negatively regulates type 1 interferon and inflammatory cytokine production by targeting TRAF6 and IRAK1 $[52,53]$. Transfection of a miR-146a mimic in THP-1 cells leads to reduction in levels of major cytokines/chemokines induced by LPS [52]. Collectively, these data suggest that increased levels of these miRNAs in circulating EVs may have protective anti-inflammatory effects during HIV pathogenesis.

We acknowledge limitations of the study, particularly those related to purity of EV preparations [54]. Although plasma is a good source of EVs, it is challenging to separate plasma EVs from abundant plasma proteins, larger microvesicles, lipoprotein particles, and ribonucleoproteins. In plasma, RNAs are associated with lipoproteins (LDL/HDL) and ribonucleoproteins, which protect extracellular RNAs against RNase-mediated degradation; these particles can be coprecipitated during EV isolation, leading to contamination of EV preparations with extravesicular RNAs. In the validation cohort, we attempted to eliminate extravesicular RNAs by Proteinase K followed by RNase A treatment. This method resulted in higher purity of EV fractions, but lower EV yield. Our study was also limited by small volumes of plasma available for EV RNA isolation, leading to low RNA input. Batch effects could be another confounding factor. Additional potential confounders include effects of smoking, HCV infection, and ART treatment, which could influence some findings $[55,56]$. In particular, given known effects of smoking on inflammation and miRNA expression and different proportions of smokers between groups in the validation cohort, we cannot exclude the possibility that smoking contributed to some of our results. Further studies in larger cohorts are needed to address the impact of cigarette smoking, HCV infection, ART drugs, and other factors on EV RNA cargo and to assess whether target genes of upregulated miRNAs identified in plasma EVs are downregulated in cells from the same individuals.

\section{Conclusions}

In conclusion, our study shows that HIV-positive individuals on ART have elevated abundance of circulating 
EVs compared to HIV-negative individuals, and these EVs carry diverse small RNA cargo including miRNA, piRNA, snRNA, tRNA, and snoRNA, with miRNAs being the most abundant. Given their functional role in post-transcriptional regulation, we focused on miRNAs for validation studies and showed that six miRNAs (miR-27b-3p, - 21-5p, - 122-5p, -10a-5p, - 423-5p, and -146a-5p) were increased in plasma EVs of HIV-positive compared to HIV-negative individuals, and these miRNAs correlated with metabolite markers of inflammation and oxidative stress. These upregulated EV miRNAs are predicted to have functional associations with oxidative stress responses, interferon gamma signaling, Toll-like receptor signaling, TGF beta signaling, and Notch signaling based on over-representation and pathway enrichment analyses. These findings suggest that circulating EV miRNAs may reflect ongoing pathophysiological processes in HIV-infected individuals on ART, serving as potential biomarkers of inflammation and oxidative stress and targetable mechanisms involved in disease pathogenesis.

\section{Methods}

\section{Study subjects}

The study was performed in accordance with guidelines in the Declaration of Helsinki. Test cohort plasma samples were obtained from HIV-positive $(n=12$, age $43-$ 60 years) and HIV-negative subjects $(n=12$, age $31-62$ years) enrolled in the Chicago site of the Multicenter AIDS Cohort Study (MACS), an ongoing prospective study of HIV-infected and -uninfected MSM. All subjects were enrolled with written informed consent and IRB approval at Northwestern University Feinberg School of Medicine. Validation cohort plasma samples were obtained from HIV-positive subjects $(n=8$, age 45-67 years) enrolled in the National NeuroAIDS Tissue Consortium (NNTC) and healthy control plasma samples $(n=8)$, were from HIV-negative donors (from Bioreclamation IVT) with informed consent and IRB approval at each NNTC study site (Manhattan HIV Brain Bank, National Neurological AIDS Bank, California NeuroAIDS Tissue Network, Texas NeuroAIDS Research Center) and Dana-Farber Cancer Institute, respectively. Subjects were tested for $\mathrm{HCV}$ and $\mathrm{HBV}$, but not HIV-2, HTLV-I/II, or other infectious agents. HIVnegative groups were frequency-matched to corresponding HIV-positive groups to achieve overall balance in distributions of age, race, cocaine use, and $\mathrm{HCV}$ infection between the HIV-negative and HIV-positive groups in test and validation cohorts. Inclusion criteria for HIVpositive subjects in both cohorts were: adults over 40 years old on ART with HIV plasma viral load undetectable or below 1000 HIV RNA copies/ml. Exclusion criteria for all subjects were testing positive for $\mathrm{HBV}$ surface antigen and/or HBV DNA.

\section{EV isolation and size measurements}

Fresh frozen plasma samples $(0.5 \mathrm{ml})$ were thawed and centrifuged for $15 \mathrm{~min}$ at $3000 \mathrm{X}$ g. Cleared plasma was incubated with thromboplastin $\mathrm{D}$ for $5 \mathrm{~min}$ at room temperature to de-fibrinate plasma, followed by centrifugation at $10,600 \times \mathrm{g}$ for $5 \mathrm{~min}$. RNAse A treatment was performed $\left(10 \mu \mathrm{g} / \mathrm{ml}\right.$, at $37^{\circ} \mathrm{C}$ for $\left.15 \mathrm{~min}\right)$ to degrade extra-exosomal RNA, followed by addition of RNAse inhibitor (150 U/ml, New England Biolabs) [57]. EV fractions were isolated using PureExo Exosome Isolation kit (101Bio, Mountain View, CA) per manufacturer's instructions. Treated plasma was mixed with sample buffer and a 1:1:1 mixture of reagent $A, B$, and $C$ was added and mixed by inverting tubes several times. The mixture was incubated at $4{ }^{\circ} \mathrm{C}$ for $1 \mathrm{~h}$ and centrifuged at $5000 \mathrm{X}$ $\mathrm{g}$ for $3 \mathrm{~min}$. The middle fluffy layer containing EVs was collected and final EV pellet was resuspended in PBS. The resulting EV fraction was passed through $0.22 \mu \mathrm{m}$ filter to remove EVs larger than exosomes. EV morphology was characterized by imaging EV fractions on a Tecnai G2 Spirit BioTWIN Transmission Electron Microscope (TEM) equipped with an AMT $2 \mathrm{k}$ CCD camera at the Harvard University TEM core. Size distribution and concentration of EV fractions was measured by nanoparticle tracking analysis (NTA) on a ZetaView instrument (Particle Metrix).

\section{Small RNA isolation, library construction, and high- throughput sequencing}

Seven hundred $\mu$ l Qiazol was added to EV fractions and frozen at $-80^{\circ} \mathrm{C}$. Small RNA was isolated using miRNeasy micro kit (Qiagen) as per manufacturer's instructions. RNA quality was assessed with Agilent BioAnalyzer using a small RNA chip. All samples were treated with T4 polynucleotide kinase (New England Biolabs) to facilitate $5^{\prime}$ hydroxyl terminus phosphate labelling and allow greater binding of adaptors during library preparation [58]. Small RNA libraries were prepared by processing 12 samples per batch $(6 \mathrm{HIV}$ positive and 6 HIV-negative), using NEBNext small RNA Library Prep kit (New England BioLabs) according to the manufacturer's instructions, except $5^{\prime}$ and $3^{\prime}$ adaptors were diluted 1:3 with nuclease-free water to reduce adaptor dimer formation. The amplified libraries were resolved on a $10 \%$ Novex TBE gel (Life technologies) and a library size range from 140 to $160 \mathrm{bp}$ (derived from adapter-ligated constructs from 21 to 40 nucleotide RNA fragments) was excised from the gel for size selection and recovered in DNA elution buffer. Average size distribution of each library was determined with the Agilent Bioanalyzer System using High Sensitivity DNA 
Analysis Kit and quantified on ABI 7900HT Fast RTPCR instrument using the KAPA Library Quantification kit. All libraries were pooled and sequenced on the Illumina NextSeq 500 platform for single read 75 cycles at the Center for Cancer Computational Biology, DanaFarber Cancer Institute.

\section{Small RNA sequence data processing and mapping}

Raw sequence data from Illumina NextSeq 500 were converted to fastq format. Small-RNAseq reads were processed and quantified using the exceRpt small RNAseq pipeline (version 4.6.2) available on the Genboree Workbench [http://www.genboree.org/] (Fig. 1). The software processes each sample independently through a cascade of read-alignment and filtering steps designed to remove likely contaminants before aligning to endogenous sequence databases. Adapters were trimmed and read quality assessed by FASTQC to filter out reads with a PHRED score lower than 30 (FASTX-Toolkit v0.0.13). Reads $<16 \mathrm{nt}$ were excluded. Likely contaminant sequences derived from laboratory or rRNA contamination were removed by mapping to the UniVec (library of common contaminant sequences maintained by the NCBI) and human ribosomal RNA (rRNA) sequences using Bowtie2. Post filtering, reads were mapped to human genome and pre-miRNA sequence databases allowing for only a single mismatched base in each alignment. First, reads were mapped to miRbase version 21, gtRNAdb, piRNABank, circBase, and snoRNA-LBME databases to assign reads to miRNAs, tRNAs, piRNAs, circular RNAs, and snoRNAs, respectively. Then, remaining sequences were annotated to gencode version 24 (hg38), which includes biotypes such as protein coding transcripts, mitochondrial rRNA, mitochondrial tRNA, small nuclear RNA, long intergenic noncoding RNA (lincRNA), pseudogenes, and miscellaneous RNA.

\section{qPCR validation of miRNAs}

To validate candidate miRNAs from sequencing data, we performed qPCR analysis of miR-10a-5p, miR-122-5p, miR-125b-5p, miR-146a-5p, miR-21-5p, miR-27b-3p, miR-423-5p, and let-7a-5p. miRNA-specific miScript Primer Assays were purchased from QIAGEN (hsa-miR-10a5p: YP00204778, hsa-miR-122-5p: YP00205664, hsa-miR125b-5p: YP00205713, hsa-miR-146a-5p: YP00204688, hsamiR-21-5p: YP00204230, hsa-miR-27b-3p: YP00205915, hsa-miR-423-5p: YP00205624, hsa-let-7a-5p: YP00205727, and spike-in control, UniSp6: YP00203954). EV fractions were isolated following the same protocol as described under 'EV isolation and size measurements' with the following modifications to eliminate extravesicular RNAs: defibrinated plasma was treated with proteinase- $\mathrm{K}(0.5 \mathrm{mg} /$ $\mathrm{ml}$ for $30 \mathrm{~min}$ at $55^{\circ} \mathrm{C}$ ) to release protein-associated RNAs
[34, 59]. Following isolation of EV fraction, PMSF (5 mM final concentration) was added to inhibit proteases. RNAse $\mathrm{A}$ and RNAse inhibitor treatment was performed on EV fractions as described in 'EV isolation and size measurements'. UniSp6 spike-in RNA was added to each sample lysate and RNA isolation was performed as described above and eluted in $14 \mu \mathrm{l}$ nuclease-free water. Equal volumes of EV RNA $(6 \mu \mathrm{l})$ from each sample were reverse transcribed using the miRCURY LNA RT Kit (Qiagen) at $42^{\circ} \mathrm{C}$ for 60 min, and then the enzyme was inactivated at $95^{\circ} \mathrm{C}$ for 5 min. After activation of the polymerase enzyme at $95^{\circ} \mathrm{C}$ for $2 \mathrm{~min}, 45 \mathrm{cycles}$ of $95^{\circ} \mathrm{C}$ for $10 \mathrm{~s}$, and $56^{\circ} \mathrm{C}$ for $60 \mathrm{~s}$ were performed using miRCURY LNA SYBR Green PCR Kit (Qiagen), on the BioRad CFX96 Real-Time System. Since qPCR was performed in multiple batches, UniSp6 spike-in was assayed in each batch to monitor variation between batches. Each miRNA was assayed in duplicate and means of $2 \mathrm{Cq}$ values were calculated. Cq values were normalized $(\Delta \mathrm{Cq})$ by the global mean to account for inter-individual sample differences.

\section{Western blot analysis}

EV fractions were lysed in lysis buffer (Triton X-100 1\%, $\mathrm{NaCl} 150 \mathrm{mM}$, sodium deoxycholate $0.5 \%$, Tris-HCL 50 $\mathrm{mM}$, SDS 0.1\%, pH 7.4) and protein content measured by BioRad DC protein assay. Fifty micrograms of protein were separated in each lane of Tris SDS polyacrylamide gels (4-12\% gradient) and transferred onto PVDF membranes. Blots were blocked with $5 \%$ milk and probed overnight at $4{ }^{\circ} \mathrm{C}$ with primary antibodies against exosome markers CD9, Alix (sc-59,140, sc-53,540, SantaCruz Biotechnology), CD81 (NB100-65805, NovusBio), Flotillin-1, Tsg101 (610,821, 612,696, BD BioScience), and Apo A1 (3710-3-1000, MabTech), followed by appropriate secondary antibodies for $1 \mathrm{~h}$; signal was developed by enhanced chemiluminescence (ECL). Images were captured using a BioRad ChemiDoc ${ }^{\mathrm{Tw}}$ Imaging System.

\section{Metabolomic profiling}

Untargeted metabolomic profiling was performed by Metabolon (Durham, NC) combining three independent platforms: ultra-high performance liquid chromatography and tandem mass spectrometry (UHLC/MS2/MS) optimized for detection of acidic metabolites, UHLC/ MS2/MS optimized for detection of basic metabolites, and gas chromatography (GC)/MS. Plasma samples $(100 \mu \mathrm{l})$ were extracted using the MicroLab STAR system and processed for analysis on the three platforms as described [12]. Samples derived from pooled experimental samples served as technical replicates, extracted water samples served as blanks, and a cocktail of standards spiked into every analyzed sample allowed instrument performance monitoring. Compounds were identified by 
automated comparison of the ion features in the experimental samples to a reference library of over 4000 chemical standard entries that included retention time, molecular weight $(\mathrm{m} / \mathrm{z})$, preferred adducts, and in-source fragments as well as associated MS spectra and curated by visual inspection for quality control using software developed at Metabolon.

\section{Functional over-representation analysis of miRNAs}

We assessed functions of differentially expressed miRNAs from HIV-positive versus HIV-negative subjects using miRNA enrichment analysis and annotation tool (miEAA; https://ccb-compute2.cs.uni-saarland.de/mieaa tool/ accessed in December 2019) [60]. MiEAA is a webbased application that offers a variety of commonly applied statistical tests such as over-representation analysis (ORA) and facilitates the functional analysis of sets of miRNAs. MiEAA performs rich functional analysis in terms of miRNA categories such as gene ontology, pathways, diseases, immune cells, and species conservation, and tests whether a category is significantly enriched (FDR adjustment) in a given miRNA set with respect to a reference using statistical tests implemented in the gene set analysis toolkit, GeneTrail.

\section{Identification of miRNA-target genes}

Predicted and validated miRNA-target relationships were assessed by the web-based multiple predictor tools mirDIP v4.1.11.1 [61], and miRWalK v3.0 [62]. These tools query a series of miRNA target predictors and show which target is predicted by one or more algorithms. We also used experimentally validated targets deposited in miRTarBase v7.0 [63]. Targets from mirDIP were filtered to include only those that were predicted by at least 20 different sources and score class was set to 'very high' (top 1\%). Targets from miRWalk were filtered to include target genes present in all three databases queried by miRWalk (TargetScan 7.2, miRDB 5.0, and miRTarBase 7.0). The final set of target genes (Supplementary Table 5) for validated miRNAs was selected based on: 1) being present in both miRDIP and miRWalk filtered lists; or 2) present in either list and experimentally validated in miRTarbase. Predicted miRNA target-gene networks were constructed using Cytoscape v3.7.2 [64].

\section{Pathway enrichment analysis}

Functional enrichment analysis of miRNA-target genes was performed with REACTOME pathways using the ClueGO v2.5.5 [65] plugin of Cytoscape 3.7.2. To identify enriched pathways in the REACTOME database, we used two-sided (enrichment/depletion) tests based on hyper-geometric distribution. Pathways with $p \leq 0.05$ were selected and Benjamini-Hochberg adjustment was used to correct $p$-values for terms and groups created by ClueGO. Kappa score threshold was set to 0.6; GO tree interval was 3-8; Leading Group was selected by 'highest significance'; \% of Group Merge was 50.

\section{Data processing and statistical analysis}

For small RNA-sequence data analysis, raw read counts obtained from the Genboree Workbench's exceRpt small RNA-seq pipeline were further analyzed using $R$ (version 3.5.2). Differential expression fold-changes and $p$-values were calculated with the DEseq2 package (version 1.22.2), adjusting for library concentration, in conjunction with adjustment for 'nuisance factors' estimated from glm residuals using the RUVseq package (version 1.16.1). Altered miRNAs or protein coding sequences with absolute fold change $>1.3$ and FDR $<0.1$ were considered significant. miRNAs with $<5$ counts per million reads (cpm) in $\geq 75 \%$ of the samples were excluded and the top 50 most abundant miRNAs were selected for DE analysis. Unsupervised hierarchical clustering using the ComplexHeatmap R package (version 2.2.0) was used to evaluate clustering in heatmaps. For metabolite profiling, metabolite data was normalized by median centering. Missing values were imputed with the lower limit of detection for a given metabolite. Pearson correlations were used to evaluate relationships between plasma metabolites and miRNA levels $(p<0.05)$. qPCR miRNA levels were compared between groups using the Mann Whitney U-test in PRISM (GraphPad) $(p<0.05)$.

\section{Supplementary Information}

The online version contains supplementary material available at https://doi. org/10.1186/s12865-020-00386-5.

\footnotetext{
Additional file 1: Supplementary Figure S1: Comparison of total read counts for each RNA biotype in HIV-positive $(n=12)$ versus HIV-negative $(n=12)$ subjects. Supplementary Figure S2. Principal component analysis of the top 50 miRNAs identified by small RNA sequencing of plasma EV RNAs from HIV-positive $(n=12)$ and HIV-negative $(n=12)$ subjects. Three HIV-negative outliers (circled in green) were excluded from downstream differential expression analysis. Supplementary Figure S3. Plasma EV isolation and purification to exclude extravesicular RNAs. EV fractions were isolated from pooled plasma of healthy control subjects $(n=3)$ using the PureExo kit. Defibrinated plasma was either untreated, or treated with RNAse A, or with Proteinase-K followed by RNAse A, to eliminate extravesicular RNAs. TEM (top), particle size distribution (bottom left), particle concentration (bottom middle), and immunoblotting for exosome markers and ApoA1 (bottom right) are shown for each treatment condition. Supplementary Figure S4. Comparison of $\mathrm{Cq}$ values of miRNAs in plasma EVs of HIV-positive and HIV-negative subjects in the validation cohort, stratified by cocaine use. Mean and SEM are shown. Significance was calculated using Mann Whitney test. ( $n=8$ HIV-positive and $n=8$ HIV-negative subjects). Supplementary Figure S5: Scatter plots showing inverse relationships between PUFA metabolites and EVassociated miRNAs. Pearson correlation coefficient and $p$-value are shown above each plot. $n=16$ subjects ( $8 \mathrm{HIV}$-positive and $8 \mathrm{HIV}$-negative). DHA docosahexaenoate (22:6n3); n3 DPA, docosapentaenoate (22:5n3); n6 DPA, docosapentaenoate (22:5n6) and EPA, eicosapentaenoate (20:5n3).
} 
Additional file 2: Supplementary Table S1 - Biotype counts of different RNA species identified by RNA-sequencing of plasma EV small RNAs from HIV-positive and HIV-negative subjects in the test cohort.

Additional file 3: Supplementary Table S2 - Read counts (normalized RPM) of all mapped mature plasma EV miRNAs in the test cohort.

Additional file 4: Supplementary Table S3 - Differential expression analysis of top 70 protein coding reads showing fold change between HIV-positive $(n=8)$ versus HIV-negative $(n=12)$ subjects in the test cohort. 70 genes were selected from a total of 14,500 genes by filtering for protein coding sequences with counts $>10$ in at least $25 \%$ samples, and not identified in a blank sample from an independent experiment.

Additional file 5: Supplementary Table S4 - Over-representation analysis of differentially expressed plasma EV-miRNAs in the validation cohort using miEAA (miRNA Enrichment Analysis and Annotation) tool.

Additional file 6: Supplementary Table S5 - Predicted and validated target genes of 6 differentially expressed plasma EV-miRNAs in the validation cohort. Shown are 61 target genes selected based on the rule: 1) present in both miRDIP and miRWalk predicted target lists; OR 2) present in either miRDIP or miRWalk predicted target lists and experimentally validated (miRTarbase).

Additional file 7: Supplementary Table S6 - Enriched pathway terms of networks associated with 61 predicted and validated target genes of 6 miRNAs upregulated in the validation cohort using ClueGo software (.xlsx)

Additional file 8: Supplementary Table S7 - Levels of plasma metabolites associated with oxidative stress, polyunsaturated fatty acid (PUFA), and tryptophan/kynurenine metabolism in HIV-negative $(n=8)$ and HIV-positive $(n=8)$ subjects in the validation cohort.

Additional file 9: Supplementary Figure $\mathbf{S 6}$ - Full-length images of blots shown in Fig. 2c.

\section{Abbreviations}

ART: Antiretroviral therapy; ALT: Alanine aminotransferase; CSF: Cerebrospinal fluid; EV: Extracellular vesicles; FDR: False discovery rate; HIV: Human immunodeficiency virus type I; K:T ratio: Kynurenine: Tryptophan ratio; lincRNA: Long intergenic noncoding RNA; miRNA: MicroRNA; mRNA: Messenger RNA; NTA: Nanoparticle tracking analysis; ORA: Overrepresentation analysis; PCA: Principal component analysis; piRNA: Piwiinteracting RNA; rRNA: Ribosomal RNA; ROS: Reactive oxygen species; snoRNA: Small nucleolar RNA; snRNA: Small nuclear RNA; TEM: Transmission electron microscopy; tRNA: transfer RNA

\section{Acknowledgements}

We thank the Center for Cancer and Computational Biology core at DanaFarber Cancer Institute for small RNA library preparation and RNA sequencing, Harvard Medical School Electron Microscopy Facility for TEM imaging, and Harvard Chan Bioinformatics core for statistical advice.

\section{Authors' contributions}

S.C. performed experiments, organized inventories, analyzed data, drafted the manuscript, performed bioinformatics and statistical analysis, and prepared figures and Tables. D.L. performed bioinformatics and statistical analysis, V.M. organized clinical data and helped with data analysis. S.M.W participated in cohort selection, study design, and data analysis. D.G. designed and supervised the study and participated in data analysis and drafting the manuscript. All authors read, participated in editing the manuscript, and approved the final manuscript.

\section{Funding}

This work was supported by National Institute on Drug Abuse (NIDA) grant R01 DA040391 to D.G. The MACS is funded by the National Institute of Allergy and Infectious Diseases (NIAID; U01-Al35039, U01-Al35040, U01Al35041, U01-Al35042, and UM1-Al35043), with additional co-funding from the National Cancer Institute (NCI), National Institute on Drug Abuse (NIDA), and National Institute of Mental Health (NIMH) at the National Institutes of Health (NIH). MACS data collection is also supported by UL1-TR000424 (JHU (TSA). NNTC sites were supported by National Institute of Mental Health and National Institute of Neurological Disorders and Stroke (grants U24MH100931,
U24MH100930, U24MH100929, U24MH100928, 24MH100925). The funding sources had no role in study design, collection and analysis of data, writing the manuscript, and decision to submit the paper for publication.

\section{Availability of data and materials}

All data generated or analyzed during this study are included in this published article (and its Supplementary Data files) or available from the corresponding author on reasonable request.

\section{Ethics approval and consent to participate}

Plasma samples in the test cohort were obtained from the Chicago site of the Multicenter AIDS Cohort Study (MACS). All participants were enrolled with written informed consent and IRB approval at Northwestern University Feinberg School of Medicine. Plasma from HIV-positive subjects in the validation cohort were obtained from National NeuroAIDS Tissue Consortium (NNTC) and healthy control plasma samples were from Bioreclamation IVT, with written informed consent from all participants and IRB approval at each NNTC study site (Manhattan HIV Brain Bank, National Neurological AIDS Bank, California NeuroAIDS Tissue Network, Texas NeuroAIDS Research Center) and Dana-Farber Cancer Institute, respectively.

\section{Consent for publication}

Not applicable.

\section{Competing interests}

The authors declare that they have no competing interests.

\section{Author details}

'Department of Cancer Immunology and Virology, Dana-Farber Cancer Institute, Boston, MA, USA. ${ }^{2}$ Division of Infectious Diseases, Department of Medicine, Northwestern University Feinberg School of Medicine, Chicago, IL, USA. ${ }^{3}$ Department of Neurology, Harvard Medical School, Boston, MA, USA.

Received: 21 June 2020 Accepted: 26 October 2020

Published online: 11 November 2020

\section{References}

1. Deeks SG, Tracy R, Douek DC. Systemic effects of inflammation on health during chronic HIV infection. Immunity. 2013;39(4):633-45.

2. Brenchley JM, Price DA, Schacker TW, Asher TE, Silvestri G, Rao S, Kazzaz Z, Bornstein $\mathrm{E}$, Lambotte $\mathrm{O}$, Altmann $\mathrm{D}$, et al. Microbial translocation is a cause of systemic immune activation in chronic HIV infection. Nat Med. 2006; 12(12):1365-71.

3. d'Ettorre G, Paiardini M, Ceccarelli G, Silvestri G, Vullo V. HIV-associated immune activation: from bench to bedside. AIDS Res Hum Retrovir. 2011; 27(4):355-64.

4. Suresh DR, Annam V, Pratibha K, Prasad BV. Total antioxidant capacity--a novel early bio-chemical marker of oxidative stress in HIV infected individuals. J Biomed Sci. 2009;16:61.

5. Masia M, Padilla S, Fernandez M, Rodriguez C, Moreno A, Oteo JA, Antela A, Moreno S, Del Amo J, Gutierrez F, et al. Oxidative stress predicts all-cause mortality in HIV-infected patients. PLoS One. 2016;11(4):e0153456.

6. Ivanov AV, Valuev-Elliston VT, Ivanova ON, Kochetkov SN, Starodubova ES, Bartosch B, Isaguliants MG. Oxidative stress during HIV infection: mechanisms and consequences. Oxidative Med Cell Longev. 2016;2016: 8910396.

7. De Toro J, Herschlik L, Waldner C, Mongini C. Emerging roles of exosomes in normal and pathological conditions: new insights for diagnosis and therapeutic applications. Front Immunol. 2015;6:203.

8. Abels ER, Breakefield XO. Introduction to extracellular vesicles: biogenesis, RNA cargo selection, content, release, and uptake. Cell Mol Neurobiol. 2016; 36(3):301-12.

9. Yu X, Odenthal M, Fries JW. Exosomes as miRNA carriers: formationfunction-future. Int J Mol Sci. 2016;17(12):2028.

10. Kadiu I, Narayanasamy P, Dash PK, Zhang W, Gendelman HE. Biochemical and biologic characterization of exosomes and microvesicles as facilitators of HIV-1 infection in macrophages. J Immunol. 2012;189(2):744-54.

11. Hubert A, Subra C, Jenabian MA, Tremblay Labrecque PF, Tremblay C, Laffont B, Provost P, Routy JP, Gilbert C. Elevated abundance, size, and MicroRNA content of plasma extracellular vesicles in Viremic HIV-1+ 
patients: correlations with known markers of disease progression. J Acquir Immune Defic Syndr. 2015;70(3):219-27.

12. Chettimada S, Lorenz DR, Misra V, Dillon ST, Reeves RK, Manickam C, Morgello S, Kirk GD, Mehta SH, Gabuzda D. Exosome markers associated with immune activation and oxidative stress in HIV patients on antiretroviral therapy. Sci Rep. 2018;8(1):7227.

13. Lenassi M, Cagney G, Liao M, Vaupotic T, Bartholomeeusen K, Cheng Y, Krogan NJ, Plemenitas A, Peterlin BM. HIV Nef is secreted in exosomes and triggers apoptosis in bystander CD4+ T cells. Traffic. 2010;11(1): 110-22.

14. Fang Y, Wu N, Gan X, Yan W, Morrell JC, Gould SJ. Higher-order oligomerization targets plasma membrane proteins and HIV gag to exosomes. PLoS Biol. 2007:5(6):e158.

15. Narayanan A, lordanskiy S, Das R, Van Duyne $R$, Santos S, Jaworski E, Guendel I, Sampey G, Dalby E, Iglesias-Ussel M, et al. Exosomes derived from HIV-1-infected cells contain trans-activation response element RNA. J Biol Chem. 2013;288(27):20014-33.

16. Konadu KA, Chu J, Huang MB, Amancha PK, Armstrong W, Powell MD, Villinger F, Bond VC. Association of Cytokines with Exosomes in the plasma of HIV-1-seropositive individuals. J Infect Dis. 2015;211(11):1712-6.

17. Khatua AK, Taylor HE, Hildreth JE, Popik W. Exosomes packaging APOBEC3G confer human immunodeficiency virus resistance to recipient cells. J Virol. 2009;83(2):512-21.

18. Yao Z, Qiao Y, Li X, Chen J, Ding J, Bai L, Shen F, Shi B, Liu J, Peng L, et al. Exosomes exploit the virus entry machinery and pathway to transmit alpha interferon-induced antiviral activity. J Virol. 2018;92(24):01578-18.

19. Boelens MC, Wu TJ, Nabet BY, Xu B, Qiu Y, Yoon T, Azzam DJ, Twyman-Saint Victor C, Wiemann BZ, Ishwaran $\mathrm{H}$, et al. Exosome transfer from stromal to breast cancer cells regulates therapy resistance pathways. Cell. 2014;159(3): 499-513.

20. Murray DD, Suzuki K, Law M, Trebicka J, Neuhaus Nordwall J, Johnson M, Vjecha MJ, Kelleher AD, Emery S. Circulating miR-122 and miR-200a as biomarkers for fatal liver disease in ART-treated, HIV-1-infected individuals. Sci Rep. 2017;7(1):10934.

21. O'Meara T, Kong Y, Chiarella J, Price RW, Chaudhury R, Liu X, Spudich S, Robertson K, Emu B, Lu L. Exosomal MicroRNAs associate with neuropsychological performance in individuals with HIV infection on antiretroviral therapy. J Acquir Immune Defic Syndr. 2019;82(5):514-22.

22. Rozowsky J, Kitchen RR, Park JJ, Galeev TR, Diao J, Warrell J, Thistlethwaite W, Subramanian SL, Milosavljevic A, Gerstein M. exceRpt: a comprehensive analytic platform for extracellular RNA profiling. Cell Syst. 2019;8(4):352-7 e353.

23. Chiang K, Sung TL, Rice AP. Regulation of cyclin T1 and HIV-1 replication by microRNAs in resting CD4+ T lymphocytes. J Virol. 2012;86(6):3244-52.

24. Piedade D, Azevedo-Pereira JM. MicroRNAs, HIV and HCV: a complex relation towards pathology. Rev Med Virol. 2016;26(3):197-215.

25. Huang J, Wang F, Argyris E, Chen K, Liang Z, Tian H, Huang W, Squires K, Verlinghieri G, Zhang H. Cellular microRNAs contribute to HIV-1 latency in resting primary CD4+ T lymphocytes. Nat Med. 2007;13(10):1241-7.

26. Whisnant AW, Bogerd HP, Flores O, Ho P, Powers JG, Sharova N, Stevenson M, Chen $\mathrm{CH}$, Cullen BR. In-depth analysis of the interaction of HIV-1 with cellular microRNA biogenesis and effector mechanisms. MBio. 2013:4(2):e000193.

27. Parikh VN, Park J, Nikolic I, Channick R, Yu PB, De Marco T, Hsue PY, Chan SY. Brief report: coordinated modulation of circulating miR-21 in HIV, HIVassociated pulmonary arterial hypertension, and HIV/hepatitis $C$ virus Coinfection. J Acquir Immune Defic Syndr. 2015;70(3):236-41.

28. Hulsmans M, De Keyzer D, Holvoet P. MicroRNAs regulating oxidative stress and inflammation in relation to obesity and atherosclerosis. FASEB J. 2011; 25(8):2515-27.

29. Rastogi N, Gara RK, Trivedi R, Singh A, Dixit P, Maurya R, Duggal S, Bhatt ML, Singh S, Mishra DP. (6)-Gingerolinduced myeloid leukemia cell death is initiated by reactive oxygen species and activation of miR-27b expression. Free Radic Biol Med. 2014;68:288-301.

30. Simionescu N, Niculescu LS, Sanda GM, Margina D, Sima AV. Analysis of circulating microRNAs that are specifically increased in hyperlipidemic and/ or hyperglycemic sera. Mol Biol Rep. 2014;41(9):5765-73.

31. Engedal N, Zerovnik E, Rudov A, Galli F, Olivieri F, Procopio AD, Rippo MR, Monsurro V, Betti M, Albertini MC. From oxidative stress damage to pathways, networks, and autophagy via MicroRNAs. Oxidative Med Cell Longev. 2018;2018:4968321.

32. Neilsen CT, Goodall GJ, Bracken CP. IsomiRs---the overlooked repertoire in the dynamic microRNAome. Trends Genet. 2012;28(11):544-9.
33. Karimi N, Cvjetkovic A, Jang SC, Crescitelli R, Hosseinpour Feizi MA, Nieuwland R, Lotvall J, Lasser C. Detailed analysis of the plasma extracellular vesicle proteome after separation from lipoproteins. Cell Mol Life Sci. 2018; 75(15):2873-86

34. Li L, Zhu D, Huang L, Zhang J, Bian Z, Chen X, Liu Y, Zhang CY, Zen K. Argonaute 2 complexes selectively protect the circulating microRNAs in cell-secreted microvesicles. PLoS One. 2012;7(10):e46957.

35. Vickers KC, Palmisano BT, Shoucri BM, Shamburek RD, Remaley AT. MicroRNAs are transported in plasma and delivered to recipient cells by high-density lipoproteins. Nat Cell Biol. 2011;13(4):423-33.

36. Fuchs D, Forsman A, Hagberg L, Larsson M, Norkrans G, Reibnegger G, Werner ER, Wachter $\mathrm{H}$. Immune activation and decreased tryptophan in patients with HIV-1 infection. J Interf Res. 1990;10(6):599-603.

37. Huengsberg M, Winer JB, Gompels M, Round R, Ross J, Shahmanesh M. Serum kynurenine-to-tryptophan ratio increases with progressive disease in HIV-infected patients. Clin Chem. 1998;44(4):858-62.

38. Seymour CW, Yende S, Scott MJ, Pribis J, Mohney RP, Bell LN, Chen YF, Zuckerbraun BS, Bigbee WL, Yealy DM, et al. Metabolomics in pneumonia and sepsis: an analysis of the GenIMS cohort study. Intensive Care Med. 2013;39(8):1423-34.

39. Kowalczyk-Pachel D, Iciek M, Wydra K, Nowak E, Gorny M, Filip M, Wlodek L, Lorenc-Koci E. Cysteine metabolism and oxidative processes in the rat liver and kidney after acute and repeated cocaine treatment. PLoS One. 2016; 11(1):e0147238.

40. Amorim MG, Valieris R, Drummond RD, Pizzi MP, Freitas VM, SinigagliaCoimbra R, Calin GA, Pasqualini R, Arap W, Silva IT, et al. A total transcriptome profiling method for plasma-derived extracellular vesicles: applications for liquid biopsies. Sci Rep. 2017;7(1):14395.

41. Yuan T, Huang X, Woodcock M, Du M, Dittmar R, Wang Y, Tsai S, Kohli M, Boardman L, Patel T, et al. Plasma extracellular RNA profiles in healthy and cancer patients. Sci Rep. 2016;6:19413.

42. El-Mogy M, Lam B, Haj-Ahmad TA, McGowan S, Yu D, Nosal L, Rghei N, Roberts P, Haj-Ahmad Y. Diversity and signature of small RNA in different bodily fluids using next generation sequencing. BMC Genomics. 2018;19(1):408.

43. Franco S, Buccione D, Pluvinet R, Mothe B, Ruiz L, Nevot M, Jordan-Paiz A, Ramos L, Ausso S, Morillas RM, et al. Large-scale screening of circulating microRNAs in individuals with HIV-1 mono-infections reveals specific liver damage signatures. Antivir Res. 2018;155:106-14.

44. Bandiera S, Pfeffer S, Baumert TF, Zeisel MB. miR-122--a key factor and therapeutic target in liver disease. J Hepatol. 2015;62(2):448-57.

45. Chen AK, Sengupta P, Waki K, Van Engelenburg SB, Ochiya T, Ablan SD, Freed EO, Lippincott-Schwartz J. MicroRNA binding to the HIV-1 gag protein inhibits gag assembly and virus production. Proc Natl Acad Sci U S A. 2014;111(26):E2676-83.

46. Castro FOF, Silva JM, Dorneles GP, Barros JBS, Ribeiro CB, Noronha I, Barbosa GR, Souza LCS, Guilarde AO, Pereira A, et al. Distinct inflammatory profiles in HIV-infected individuals under antiretroviral therapy using cannabis, cocaine or cannabis plus cocaine. AIDS. 2019;33(12):1831-42.

47. Cisneros IE, Erdenizmenli M, Cunningham KA, Paessler S, Dineley KT. Cocaine evokes a profile of oxidative stress and impacts innate antiviral response pathways in astrocytes. Neuropharmacology. 2018;135:431-43.

48. Xiao J, Pan Y, Li XH, Yang XY, Feng YL, Tan HH, Jiang L, Feng J, Yu XY. Cardiac progenitor cell-derived exosomes prevent cardiomyocytes apoptosis through exosomal miR-21 by targeting PDCD4. Cell Death Dis. 2016;7(6):e2277.

49. Thulasingam S, Massilamany C, Gangaplara A, Dai H, Yarbaeva S, Subramaniam S, Riethoven JJ, Eudy J, Lou M, Reddy J. miR-27b*, an oxidative stress-responsive microRNA modulates nuclear factor-kB pathway in RAW 264.7 cells. Mol Cell Biochem. 2011;352(1-2):181-8.

50. Whisnant AW, Kehl T, Bao Q, Materniak M, Kuzmak J, Lochelt M, Cullen BR. Identification of novel, highly expressed retroviral microRNAs in cells infected by bovine foamy virus. J Virol. 2014;88(9):4679-86.

51. Taganov KD, Boldin MP, Chang KJ, Baltimore D. NF-kappaB-dependent induction of microRNA miR-146, an inhibitor targeted to signaling proteins of innate immune responses. Proc Natl Acad Sci U S A. 2006;103(33):12481-6.

52. Pauley KM, Satoh M, Pauley BA, Dominguez-Gutierrez PR, Wallet SM, Holliday LS, Cha S, Reeves WH, Chan EK. Formation of GW/P bodies as marker for microRNA-mediated regulation of innate immune signaling in THP-1 cells. Immunol Cell Biol. 2010;88(2):205-12.

53. Hou J, Wang P, Lin L, Liu X, Ma F, An H, Wang Z, Cao X. MicroRNA-146a feedback inhibits RIG-I-dependent type I IFN production in macrophages by targeting TRAF6, IRAK1, and IRAK2. J Immunol. 2009;183(3):2150-8. 
54. Geyer PE, Kulak NA, Pichler G, Holdt LM, Teupser D, Mann M. Plasma proteome profiling to assess human health and disease. Cell Syst. 2016;2(3): 185-95.

55. Longatti $A$. The dual role of Exosomes in hepatitis a and $C$ virus transmission and viral immune activation. Viruses. 2015;7(12):6707-15.

56. Petoumenos K, Law MG. Smoking, alcohol and illicit drug use effects on survival in HIV-positive persons. Curr Opin HIV AIDS. 2016;11(5):514-20.

57. Huang X, Yuan T, Tschannen M, Sun Z, Jacob H, Du M, Liang M, Dittmar RL, Liu Y, Liang M, et al. Characterization of human plasma-derived exosomal RNAs by deep sequencing. BMC Genomics. 2013;14:319.

58. Danielson KM, Rubio R, Abderazzaq F, Das S, Wang YE. High throughput sequencing of extracellular RNA from human plasma. PLoS One. 2017;12(1): e0164644.

59. Arroyo JD, Chevillet JR, Kroh EM, Ruf IK, Pritchard CC, Gibson DF, Mitchell PS, Bennett CF, Pogosova-Agadjanyan EL, Stirewalt DL, et al. Argonaute2 complexes carry a population of circulating microRNAs independent of vesicles in human plasma. Proc Natl Acad Sci U S A. 2011;108(12):5003-8.

60. Backes C, Khaleeq QT, Meese E, Keller A. miEAA: microRNA enrichment analysis and annotation. Nucleic Acids Res. 2016;44(W1):W110-6.

61. Tokar T, Pastrello C, Rossos AEM, Abovsky M, Hauschild AC, Tsay M, Lu R, Jurisica I. mirDIP 4.1-integrative database of human microRNA target predictions. Nucleic Acids Res. 2018;46(D1):D360-70.

62. Sticht C, De La Torre C, Parveen A, Gretz N. miRWalk: An online resource for prediction of microRNA binding sites. PLoS One. 2018;13(10):e0206239.

63. Chou CH, Shrestha S, Yang CD, Chang NW, Lin YL, Liao KW, Huang WC, Sun TH, Tu SJ, Lee WH, et al. miRTarBase update 2018: a resource for experimentally validated microRNA-target interactions. Nucleic Acids Res. 2018;46(D1):D296-302.

64. Shannon P, Markiel A, Ozier O, Baliga NS, Wang JT, Ramage D, Amin N, Schwikowski B, Ideker T. Cytoscape: a software environment for integrated models of biomolecular interaction networks. Genome Res. 2003;13(11): 2498-504.

65. Bindea G, Mlecnik B, Hackl H, Charoentong P, Tosolini M, Kirilovsky A, Fridman WH, Pages F, Trajanoski Z, Galon J. ClueGO: a Cytoscape plug-in to decipher functionally grouped gene ontology and pathway annotation networks. Bioinformatics. 2009;25(8):1091-3.

\section{Publisher's Note}

Springer Nature remains neutral with regard to jurisdictional claims in published maps and institutional affiliations.

Ready to submit your research? Choose BMC and benefit from:

- fast, convenient online submission

- thorough peer review by experienced researchers in your field

- rapid publication on acceptance

- support for research data, including large and complex data types

- gold Open Access which fosters wider collaboration and increased citations

- maximum visibility for your research: over $100 \mathrm{M}$ website views per year

At BMC, research is always in progress.

Learn more biomedcentral.com/submissions 